\title{
Thermodynamics of $d$-dimensional Schwarzschild black holes in the canonical ensemble
}

\author{
Rui André $\oplus^{*}$ and José P. S. Lemos $\oplus^{\dagger}$ \\ Centro de Astrofísica e Gravitação—CENTRA, Departamento de Física, Instituto Superior Técnico—IST, \\ Universidade de Lisboa-UL, Av. Rovisco Pais 1, 1049-001 Lisboa, Portugal
}

(Received 9 December 2020; accepted 12 February 2021; published 25 March 2021)

We study the thermodynamics of a $d$-dimensional Schwarzschild black hole, also known as a Schwarzschild-Tangherlini black hole, in the canonical ensemble. This generalizes York's formalism, which has been initially applied to four dimensions and later to five dimensions, to any number $d$ of dimensions. The canonical ensemble, characterized by a cavity of fixed radius $r$ and fixed temperature $T$ at the boundary, allows for two possible black hole solutions in thermal equilibrium, a smaller black hole and a larger black hole. In four and five dimensions, these solutions have a direct exact form, whereas in an arbitrary number of dimensions, one is compelled to resort to approximation schemes or numerical calculations. From the Euclidean action and the path integral approach, we obtain the free energy, the thermodynamic energy, the thermodynamic pressure, and the entropy, of the black hole plus cavity system. The entropy of the system is given by the Bekenstein-Hawking area law. The analysis of the heat capacity of the system shows that the smaller black hole is in unstable equilibrium and the larger black hole is in stable equilibrium. The $d$-dimensional photon sphere radius divides the stability criterion. Indeed, if the cavity's radius is larger than the photon sphere radius, and so the black hole is small, the system is unstable, if the cavity's radius is smaller than the photon sphere radius, and so the black hole is large, the system is stable. To study perturbations on the system, a generalized free energy function is obtained that also allows one to understand the possible phase transitions between classical hot flat space and the black holes. The Buchdahl radius, that appears naturally in the general relativistic study of star structure, also shows up in our context; the free energy is zero when the cavity's radius has the $d$-dimensional Buchdahl radius value. Then, if the cavity's radius is larger than the Buchdahl radius, classical hot flat space phase cannot make a phase transition to a black hole phase, and if smaller, classical hot flat space can nucleate a black hole. The roles of both the photon sphere and the Buchdahl limit are present for every dimension $d$, indicating that, besides their known role in dynamics, these radii also play a role in the thermodynamics of gravitational systems. The close link between the canonical analysis performed and the direct perturbation of the path integral is also pointed out. Since hot flat space is a quantum system made purely of gravitons, if only gravitation is considered, it is of great interest to compare the $d$-dimensional free energies of quantum hot flat space and the stable black hole to find for which ranges of $r$ and $T$, the quantities that characterize the canonical ensemble, one phase predominates over the other. Phase diagrams for a few different dimensions are displayed. The density of states at a given energy is found through an inverse Laplace transformation giving back the entropy of the stable black hole. Several side calculations and further deliberations are performed, namely, the calculation for the approximate expressions for the canonical ensemble black hole horizon radii, a brief study of the photon orbit radius and the Buchdahl radius in the $d$-dimensional Schwarzschild solution, a connection to the thermodynamics of thin shells in $d$ spacetime dimensions which are systems that are also apt to a rigorous thermodynamic study, a presentation of quantum hot flat space in $d$ spacetime dimensions as a thermodynamic system, an analysis of classical hot flat space in $d$ spacetime dimensions as a product of quantum hot flat space with the black hole transitions and the corresponding phase diagrams for a few different dimensions, and a synopsis with the relevance of the work. It is still worth mentioning that the comparison of the thermodynamics of $d$-dimensional Schwarzschild black holes and classical hot flat space in the canonical ensemble with the thermodynamics

*rui.andre@tecnico.ulisboa.pt

joselemos@tecnico.ulisboa.pt

Published by the American Physical Society under the terms of the Creative Commons Attribution 4.0 International license. Further distribution of this work must maintain attribution to the author(s) and the published article's title, journal citation, and DOI. Funded by $S C O A P^{3}$. 
of spherical thin shells in $d$ dimensions yields a striking direct matching between the two systems, most notably that the photon sphere radius appears here as a thermodynamic stability divisor in both systems, and the Buchdahl radius that appears on thermodynamic grounds for canonical black holes appears also as a thermodynamic and as a dynamical radius for thin shells.

DOI: 10.1103/PhysRevD.103.064069

\section{INTRODUCTION}

Black holes are physical systems that possess thermodynamic properties. The path-integral approach to quantum gravity is a powerful technique that when applied to black holes displays them clearly as thermodynamic systems. In this approach, the geometry of a four-dimensional Schwarzschild black hole, say, is Euclideanized, and its temperature is fixed by the correct period in the imaginary time, putting the black hole in a state of equilibrium with a heat bath at the prescribed temperature, the Hartle-Hawking vacuum state [1]. The black hole entropy $S$ can then be found to be $S=\frac{1}{4} A_{+}$in Planck units, where area $A_{+}$is the event horizon area. This entropy stems from the contribution of the classical Euclidean Einstein action of the black hole metric to the partition function, and its cause is the nontrivial topology with a nonzero Euler characteristic of the Euclidean four-dimensional Schwarzschild black hole, in contrast to ordinary quantum field theories, where the classical contribution to the action is absorbed into the normalization of the functional integral [2]. It is of great interest to extend this approach to $d$-dimensional Schwarzschild black holes. The generalization of the Hartle-Hawking vacuum setting to $d$-dimensional Schwarzschild black holes has been done in Ref. [3]. Moreover, the understanding that the black hole entropy in $d$ dimensions, with $d \geq 4$, comes from topological considerations, specifically, the Euler characteristic of the twodimensional plane spanned by the Euclidean time and radial spatial coordinate, was performed in Ref. [4].

With the path-integral approach in hand, York understood that the correct setting to study black thermodynamics, in particular a four-dimensional Schwarzschild black hole, was to work with the canonical ensemble of statistical mechanics [5] which provides a complete description of the thermodynamics of those systems. In the canonical ensemble, the black hole is placed inside a cavity whose boundary has radius $r$ and is at temperature $T$; i.e., the cavity is in thermal equilibrium with a heat bath at temperature $T$. The Euclidean action for the system shows that the instanton solution admits two boundary configurations; i.e., there are two black hole solutions for the canonical boundary data. One solution yields a small black hole inside a large cavity in thermal, but unstable, equilibrium, which was the system studied in great detail in Ref. [6] that had been previously studied in Refs. [1,2]. The other solution yields a large black hole inside a cavity with a size of the same order of the black hole, in thermal and stable equilibrium, which was studied in Ref. [7]. By using the canonical ensemble, and showing there are stable configurations, the thermodynamics of black holes is then unified with a proper setting. The canonical ensemble path-integral approach can be extended to more complex systems, as has been done for electrically charged black holes in the grand canonical ensemble [8], for black holes in anti-de Sitter spacetimes [9], and even for matter configurations [10] or matter plus black hole systems [11]. In higher dimensions, York's formalism can also be developed. The five-dimensional Schwarzschild black hole has shown to be of particular interest, because the exact solutions for the instantons take a simple form which allows for an approach with fewer approximations than those used originally, with the smaller unstable solution and the larger stable solution being found exactly [12]. Moreover, the stable and unstable thermodynamic modes of a $d$-dimensional Schwarzschild black hole have been studied in detail in Ref. [13]; see also Refs. [14,15]. In this work, we generalize the four- and fivedimensional canonical ensemble path integral approach for a spherical symmetric black hole in $d$ spacetime dimensions, enabling us to extract intrinsic features that might arise.

Now, another gravitational system that can be handled in pure thermodynamic grounds is a spherical thin shell that separates a Minkowski interior from some exterior spacetime. Fixing the temperature on the shell, and given a wellprescribed first law of thermodynamics at the shell, a powerful thermodynamic formalism can be developed that gives the entropy and the stability of the shell. In four dimensions, for a shell with a Schwarzschild exterior, the problem was treated in Ref. [16], and for a shell with a Reissner-Nordström exterior, the problem was treated in Ref. [17]. The study of thermodynamics of thin shells in $d$ dimensions with a Schwarzschild exterior was solved in Ref. [18]. We are thus led to compare here the $d$-dimensional black hole in the canonical ensemble studied in this work with the $d$-dimensional thin matter shells in the thermodynamic setting studied in Ref. [18].

There are some results that will be used. In four dimensions, the solution we are interested in is the Schwarzschild solution. An analysis on the quasilocal energy of spherical spacetimes that has applications in thermodynamic problems was done in Ref. [19]. The photonic radius, the radius where the photons have circular orbits, in Schwarzschild in four dimensions is given by 
$r=\frac{3}{2} r_{+}$where $r_{+}$is the gravitational radius, and since $r_{+}=2 m$, one also can write $r=3 m$, where $m$ is the spacetime mass. This special radius also appears in the thermodynamic study of the black hole in the canonical ensemble, as York noticed. The Buchdahl radius, i.e., the radius for the maximum compactness of a general relativistic star [20], or of a general relativistic thin shell under certain conditions [21], is given by $r=\frac{9}{8} r_{+}$where $r_{+}$is the gravitational radius, and since $r_{+}=2 m$, one also can write $r=\frac{9}{4} m$, where again $m$ is the spacetime mass. This special radius also appears in the thermodynamic study of the black hole in the canonical ensemble, as we noticed here. When studying the black hole in the canonical ensemble, one also needs the thermodynamic properties of a radiation gas in four spacetime dimensions as given in any book in thermodynamics. In higher $d$ dimensions, the solution we are interested in is the $d$-dimensional Schwarzschild solution [22], also called Schwarzschild-Tangherlini or simply Tangherlini solution. Quasilocal energy on higher-dimensional spacetimes has not been performed, but certainly the results are maintained. The photonic radius in Schwarzschild in $d$ dimensions is given in Ref. [23]. This special radius also appears in the thermodynamic study of the $d$-dimensional black hole in the canonical ensemble. The Buchdahl radius for most compactness of a $d$-dimensional star is given in Ref. [24] and for a shell in a $d$-dimensional spacetime we give here. This special radius also appears in the thermodynamic study of the black hole in the canonical ensemble, as we noticed here. When studying the black hole in the canonical ensemble, one needs to use the thermodynamic properties of a radiation gas in $d$ spacetime dimensions as given in Ref. [25].

The paper is organized as follows. In Sec. II, we prepare the cavity at a fixed radius $r$ and temperature $T$ at the cavity's wall. Inside the cavity, for the $d$-dimensional Schwarzschild metric, we look for the black hole solutions which satisfy thermal equilibrium with the cavity's wall. The section is split in two parts, where we find an expression for the smaller black hole first, followed by the larger one. We also derive the Euclidean EinsteinHilbert action for a $d$-dimensional Schwarzschild black hole as a function of the cavity's radius and temperature. In Sec. III, from the action, we derive all the thermodynamic quantities associated to the black hole plus cavity system, particularly, the thermodynamic energy, pressure, and entropy, along with the first law of thermodynamics for the system. In Sec. IV, we find the heat capacity for the system, which is crucial in identifying the thermal stability of the solutions. In Sec. V, with the free energy function being the thermodynamic potential of the canonical ensemble proportional to the action, we can better interpret possible state transitions inside the cavity, discussing the possibility of black hole nucleation, or even the transition from a black hole state to flat space. In Sec. VI, we address and comment on the relationship between the action functional to second order and thermodynamics and thermal stability. In Sec. VII, we directly compare the free energy of $d$-dimensional quantum hot flat space with the free energy of the stable black hole. With this, we can identify the conditions for each of these states being the ground state of the canonical ensemble, i.e., with the lowest free energy, or when the ground state is a superposition of both, when they have the same free energy. In Sec. VIII, we compute the density of states from the partition function for the stable black hole solution, which in turn leads to an alternative way of reproducing the area law for the entropy. In Appendix A, we develop some side calculations. In Appendix B, we dwell on two important radii that appear in the canonical ensemble context, the photon orbit radius and the Buchdahl radius. In Appendix C, we establish the relationship between the thermodynamics of black holes in a cavity in $d$ dimensions and the thermodynamics of thin matter shells in $d$ dimensions. In Appendix $\mathrm{D}$, we derive the generalized $d$-dimensional free energy and action for quantum hot flat space, along with the thermodynamic quantities used. In Appendix E, we study classical hot flat space in $d$ spacetime dimensions as the product of quantum hot flat space and analyze the corresponding black hole phase transitions for classical hot flat space. In Appendix F, we present a synopsis and further additions.

\section{CANONICAL ENSEMBLE FOR A CAVITY WITH A BLACK HOLE INSIDE: TEMPERATURE, THE EUCLIDEAN EINSTEIN ACTION, AND THE ACTION FUNCTIONAL OR PARTITION FUNCTION FOR A $d$-DIMENSIONAL SCHWARZSCHILD BLACK HOLE}

\section{A. Cavity in $d$ dimensions and the canonical temperature}

\section{Generics and temperature of the canonical ensemble}

In the canonical ensemble of a spherical symmetric thermodynamic system, we fix the radius $r$ of the cavity's boundary and the local temperature $T$ at the cavity's boundary. We also define the inverse temperature $\beta=\frac{1}{T}$, which is a useful parameter, so that the independent variables we will work with can either be $T$ and $r$ or $\beta$ and $r$. Throughout the paper, we set the speed of light $c$, the gravitational constant $G$, the Planck constant $\hbar$, and the Boltzmann constant $k_{B}$ to unity, i.e., $c=1, G=1, \hbar=1$, and $k_{B}=1$. As a consequence, the Planck length is given by $l_{P}=1$, and the Planck temperature is given by $T_{P}=1$.

The black hole solution inside the cavity follows from the $d$-dimensional Schwarzschild solution with line element given by

$$
d s^{2}=\left(1-\frac{r_{+}{ }^{d-3}}{r^{d-3}}\right) d t^{2}+\frac{d r^{2}}{1-\frac{r_{+}^{d-3}}{r^{d-3}}}+r^{2} d \Omega_{d-2}^{2},
$$


where $t$ is Euclidean time, $r$ is the coordinate radius, and $d \Omega_{d-2}^{2}=d \theta_{1}^{2}+\sum_{k=2}^{d-2}\left(\prod_{j=1}^{k-1} \sin ^{2} \theta_{j}\right) d \theta_{k}^{2}$ is the line element on the $(d-2)$-sphere, with the $\theta_{k}$ being its angles. We are using the symbol $r$ with two different meanings. One $r$ is the coordinate radius $r$ of Eq. (1). The other $r$ is the cavity's radius $r$. The coordinate radius $r$ will disappear soon and will not be mentioned anymore, so there is no possibility of confusion. In $d$ dimensions, the gravitational radius, being also the event horizon radius when there is a black hole, $r_{+}$, and the spacetime mass $m$, sometimes called the ADM mass, are related by $r_{+}^{d-3}=\frac{16 \pi}{(d-2) \Omega_{d-2}} m$, where $\Omega_{d-2}=\frac{2 \pi^{\frac{d-1}{2}}}{\Gamma\left(\frac{d-1}{2}\right)}$ is the solid angle in a spherical $d$-dimensional spacetime. Clearly, from Eq. (1), we have to impose $d \geq 4$, so that the canonical ensemble here is valid for a four- or higherdimensional spacetime.

The Euclidean metric in Eq. (1) describes the spacetime of an Euclidean black hole outside the horizon, i.e., the coordinate $r$ obeys $r \geq r_{+}$, provided that the conical singularity at $r=r_{+}$is removed by setting the correct time period to $t$. By redefining the coordinate $r$ as $r=r_{+}+\varepsilon$, with $\varepsilon$ a radial variable such that $\varepsilon \ll r_{+}$, and introducing then a new radial coordinate $\rho=\sqrt{\frac{4 r_{+} \varepsilon}{d-3}}$, the metric given in Eq. (1) reduces to $d s^{2}=$ $d \rho^{2}+\rho^{2}\left(\frac{2 r_{+}}{d-3}\right)^{2} d t^{2}$. So, in order to have no conical singularities, $t$ must have a period, which we will denote by $\beta_{\infty}$, given by $\beta_{\infty}=\frac{4 \pi r_{+}}{d-3}$. This $\beta_{\infty}$ is the inverse Hawking temperature. So, the Hawking temperature $T_{H}$, i.e., the temperature at infinity for $d$-dimensional black holes, is $T_{H}=\frac{1}{\beta_{\infty}}=\frac{d-3}{4 \pi r_{+}}$. Now, the Tolman temperature says that the temperature at some position $r$ is the temperature at infinity blueshifted to $r$. From now on $r$ denotes always the radius of the cavity. So, in order that there is thermal equilibrium between the black hole and the cavity at $r$, the temperature, or its inverse $\beta$, at $r$, must satisfy the Tolman formula. Therefore, $\beta=\beta_{\infty} \sqrt{1-\frac{r_{+}^{d-3}}{r^{d-3}}}$, or using $\beta_{\infty}=\frac{4 \pi r_{+}}{d-3}$ we obtain

$$
\beta=\frac{4 \pi r_{+}}{d-3} \sqrt{1-\frac{r_{+}^{d-3}}{r^{d-3}}} .
$$

Since

$$
T=\frac{1}{\beta},
$$

in terms of $T$, Eq. (2) is

$$
\left(\frac{r_{+}}{r}\right)^{d-1}-\left(\frac{r_{+}}{r}\right)^{2}+\left(\frac{d-3}{4 \pi r T}\right)^{2}=0 .
$$

Equation (4) is a polynomial equation with its order set by $d$. Exact solutions for $d=4$ and $d=5$ were obtained in
Refs. [5,12], respectively. In general, for $d \geq 6$, one is compelled to resort to approximation schemes or numerical calculations to solve Eq. (4), although in some dimensions, an exact, although contrived, analysis might be performed, noting that for odd $d$ Eq. (4) can have its order reduced by solving for $\left(\frac{r_{+}}{r}\right)^{2}$.

To deal with Eq. (4), we note that the cavity radius $r$ has range $r_{+} \leq r<\infty$, i.e., $0 \leq \frac{r_{+}}{r} \leq 1$. Let us also write the left-hand side of Eq. (4) as a function $f\left(\frac{r_{+}}{r}\right)$ such that $f\left(\frac{r_{+}}{r}\right)=\left(\frac{r_{+}}{r}\right)^{d-1}-\left(\frac{r_{+}}{r}\right)^{2}+\left(\frac{d-3}{4 \pi r T}\right)^{2}$. Then, at $\frac{r_{+}}{r}=0$, one has $f(0)=\left(\frac{d-3}{4 \pi r T}\right)^{2}$, and at $\frac{r_{+}}{r}=1$, one has $f(1)=\left(\frac{d-3}{4 \pi r T}\right)^{2}$, so the extreme points of the interval have the same positive value. From the first derivative of $f\left(\frac{r_{+}}{r}\right)$, one finds that it has a unique extremum, in fact a minimum, at $\frac{r_{+}}{r}=\left(\frac{2}{d-1}\right)^{\frac{1}{d-3}}$, so that $f_{\min }=f\left(\left(\frac{2}{d-1}\right) \frac{1}{d-3}\right)$. Moreover, the second derivative at this minimum of $f\left(\frac{r_{+}}{r}\right)$ is always positive. So, $f\left(\frac{r_{+}}{r}\right)$ starts at $\frac{r_{+}}{r}=0$ with value $f(0)=\left(\frac{d-3}{4 \pi r T}\right)^{2}$ positive; decreases up to $\frac{r_{+}}{r}=\left(\frac{2}{d-1}\right) \frac{1}{d-3}$, where it has a minimum value $f_{\min }=f\left(\left(\frac{2}{d-1}\right)^{\frac{1}{d-3}}\right)$; and increases back up to $\frac{r_{+}}{r}=1$ with value $f(0)=\left(\frac{d-3}{4 \pi r T}\right)^{2}$ positive. Clearly, there are solutions to Eq. (4) only if $f_{\min }=f\left(\left(\frac{2}{d-1}\right)^{\frac{1}{d-3}}\right) \leq 0$. Since Eq. (4) has only one minimum, there will be in general two solutions that degenerate into one only when the equality in the latter equation holds.

In brief, Eq. (4) only has solutions if $f_{\min }\left(\frac{r_{+}}{r}\right) \leq 0$, i.e., $f\left(\left(\frac{2}{d-1}\right)^{\frac{1}{d-3}}\right) \leq 0$. So, the condition for the canonical ensemble at fixed $r$ and $T$ to have black hole solutions $\frac{r_{+}}{r}$ is from Eq. (4),

$$
\pi r T \geq \frac{d-3}{4}\left[\left(\frac{2}{d-1}\right)^{\frac{2}{d-3}}-\left(\frac{2}{d-1}\right)^{\frac{d-1}{d-3}}\right]^{-1 / 2}
$$

There will indeed be two possible black hole solutions, $\frac{r_{+1}}{r}$ and $\frac{r_{+2}}{r}$, and when the equality holds, there is only one black hole solution, $\frac{r_{+1}}{r}=\frac{r_{+2}}{r}$.

Let us see some further properties of Eq. (5). Equation (5) gives that the minimum value that $\pi r T$ can take is given by the number of dimensions only, a property that can be clearly seen when one treats the $d$-dimensional case generically. Equation (5) also shows that as $d$ increases, the minimum value of $\pi r T$ also increases. Indeed, for $d=4$, the threshold value for the existence of a black hole is $\pi r T=\frac{3 \sqrt{3}}{8}$, or $\pi r T=0.650$ approximately. For $d=5$, the threshold value for the existence of a black holes is $\pi r T=1$. For $d \geq 6$, Eq. (5) gives that the threshold value is always larger than 1. Given Eq. (5), we need from Eq. (4) to find an expression for the two black hole solutions, i.e., for $\frac{r_{+1}}{r}$ and $\frac{r_{+2}}{r}$. Clearly, for $\pi r T \gg 1$, Eq. (4) reduces to $\left(\frac{r_{+}}{r}\right)^{d-1}-\left(\frac{r_{+}}{r}\right)^{2}=0$, so in this case, the two black hole solutions will be expansions around $\frac{r_{+}}{r}=0$ 
and $\frac{r_{+}}{r}=1$. We now turn to find approximate solutions for $r_{+1}$ and $r_{+2}$.

\section{Smaller black hole solution $r_{+1}$ and the larger black hole solution $\boldsymbol{r}_{+2}$}

Smaller black hole solution $r_{+1}$.-To find the smaller black hole solution $\frac{r_{+1}}{r}$ around $\frac{r_{+}}{r}=0$, we make a Taylor expansion and write $\frac{r_{+1}}{r}=\frac{r_{+1}}{r}(\pi r T)$ as $\frac{r_{+1}}{r}(\pi r T)=$ $\frac{a_{1}}{\pi r T}+\frac{a_{2}}{(\pi r T)^{2}}+\ldots$, where the $a_{i}$ are constants to be determined. Equating carefully power by power this expansion in Eq. (4), one finds, see Appendix A,

$r_{+1}=r\left(\frac{d-3}{4 \pi r T}+\frac{1}{2}\left(\frac{d-3}{4 \pi r T}\right)^{d-2}+\mathcal{O}\left(\frac{1}{(\pi r T)^{d-1}}\right)\right)$.

This is the smaller black hole solution $\frac{r_{+1}}{r}$ for large $T$.

Larger black hole solution $r_{+2}$.-To find the larger black hole solution $\frac{r_{+2}}{r}$ around $\frac{r_{+}}{r}=1$, we make a Taylor expansion and write $\frac{r_{+2}}{r}=\frac{r_{+2}}{r}(\pi r T)$ as $\frac{r_{+2}}{r}(\pi r T)=$ $1+\frac{b_{1}}{\pi r T}+\frac{b_{2}}{(\pi r T)^{2}}+\ldots$, where the $b_{i}$ are constants to be determined. Equating carefully power by power this expansion in Eq. (4) one finds, see Appendix A,

$$
r_{+2}=r\left(1-\frac{d-3}{16(\pi r T)^{2}}+\mathcal{O}\left(\frac{1}{(\pi r T)^{4}}\right)\right) .
$$

This is the larger black hole solution $\frac{r_{+2}}{r}$ for large $T$.

Equal radius black hole solution $r_{+1}=r_{+2}$.- -Now, there is a $T$, not large where the two black holes have equal horizon radii. This happens when the equality in Eq. (5) holds, i.e., $\pi r T=\frac{d-3}{4}\left[\left(\frac{2}{d-1}\right)^{\frac{2}{d-3}}-\left(\frac{2}{d-1}\right)^{\frac{d-1}{d-3}}\right]^{-1 / 2}$. In this case, there is only one black hole solution for Eq. (4), namely,

$$
\frac{r_{+1}}{r}=\frac{r_{+2}}{r}=\left(\frac{2}{d-1}\right)^{\frac{1}{d-3}}
$$

This means that the cavity's radius $r$ is located at the black hole's photon sphere, since the photon sphere radius is given by $r_{\mathrm{ph}}=\left(\frac{d-1}{2}\right)^{\frac{1}{d-3}} r_{+}$; see Ref. [23] for the black hole photon sphere in $d$ dimensions, and see also Appendix B.

Full solution for $r_{+1}$ and $r_{+2}$.- - In Fig. 1, the full solution of Eq. (4) is drawn displaying $r_{+1}$ and $r_{+2}$ as a function of $\pi r T$. The details are dependent on the dimension $d$ of the spacetime, but the main features are as shown.

\section{Location and the area of the cavity}

Another important characterization of the canonical ensemble, besides its temperature, is its location given by the radius $r$ of the cavity's boundary. In some instances, it is preferable to work with the cavity's boundary area $A$, which can be given in terms of $r$ as

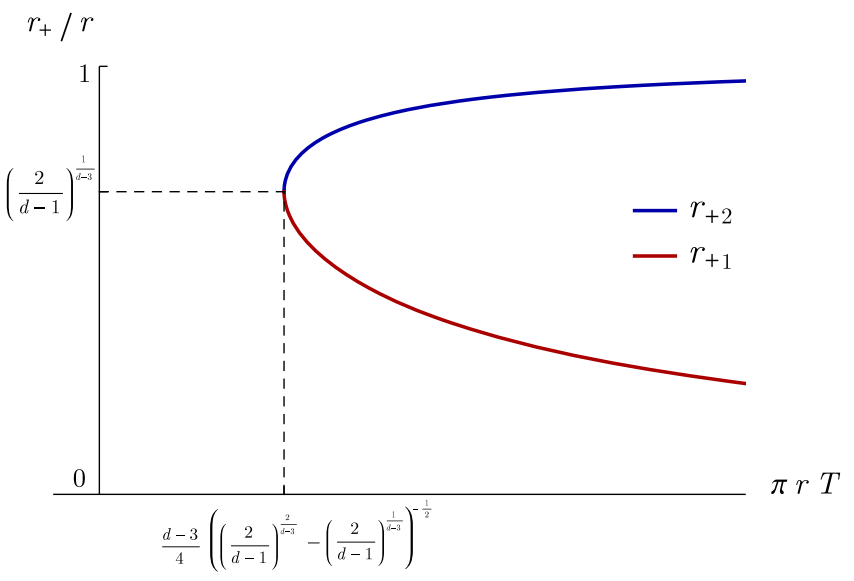

FIG. 1. The two black hole solutions $r_{+1}$ and $r_{+2}$ are shown in a plot $\frac{r_{+1}}{r}$ and $\frac{r_{+2}}{r}$, with $r$ being the cavity radius, as a function of $\pi r T$, with $\pi r T$ obeying $\pi r T \geq \frac{d-3}{4}\left[\left(\frac{2}{d-1}\right)^{\frac{2}{d-3}}-\left(\frac{2}{d-1}\right)^{\frac{d-1}{d-3}}\right]^{-1 / 2}$. The two solutions coincide when $\frac{r_{+1}}{r}=\frac{r_{+2}}{r}=\left(\frac{2}{d-1}\right)^{\frac{1}{d-3}}$, represented by a point at the intersection of the dashed lines. The corresponding radius $r$ is the radius of the photon sphere of a $d$-dimensional black hole, $r_{\mathrm{ph}}=\left(\frac{d-1}{2}\right)^{\frac{1}{d-3}} r_{+}$.

$$
A=\Omega_{d-2} r^{d-2},
$$

with $\Omega_{d-2}$ being the solid angle in a spherical $d$ dimensional spacetime.

\section{B. Euclidean-Einstein action and the action functional or the partition function for a $d$-dimensional Schwarzschild black hole}

In the path integral approach to quantum gravity, i.e., the Hartle-Hawking approach, integration of the Euclidean Einstein action over the space of metrics $g$ yields the canonical partition function $Z=\int \mathcal{D}[\mathrm{g}] \exp (-I[\mathrm{~g}])$ Taking a black hole solution as the background metric, the leading term in the expansion will be that of the classical action, specifically,

$$
Z=\mathrm{e}^{-I} \text {. }
$$

Here, $I$ is the Euclidean Einstein action of the gravitational system, being the black hole action if the system contains a black hole.

For a $d$-dimensional spacetime, the Euclidean Einstein action $I$ is

$I=-\frac{1}{16 \pi} \int_{\mathcal{M}} d^{d} x \sqrt{|g|} R-\frac{1}{8 \pi} \int_{\partial \mathcal{M}} d^{d-1} x \sqrt{|h|}[K]$,

where $\mathcal{M}$ is a compact region of the spacetime and $\partial \mathcal{M}$ is its boundary, $|g|$ is the determinant of the $d$-dimensional spacetime metric $g_{a b}, R$ is the corresponding Ricci scalar, $|h|$ is the determinant of the $(d-1)$-dimensional induced metric on the boundary, and $[K]$ is the difference of the 
extrinsic curvature $K$ on the boundary to the extrinsic curvature of an equivalent boundary embedded in flat space, $K_{\text {flat }}$. This subtraction is needed in order to normalize the action and the energy of the ensemble.

Given that we are interested in a vacuum solution, $R=0$, the action of Eq. (11) reduces to the boundary term. For the metric Eq. (1), the line element on the boundary $\partial \mathcal{M}$ for $r=\mathrm{constant}$ is $\left.d s^{2}\right|_{\partial \mathcal{M}}=\left(1-\frac{r_{-}^{d-3}}{r^{d-3}}\right) d t^{2}+r^{2} d \Omega_{d-2}^{2}$. Writing $\left.d s^{2}\right|_{\partial \mathcal{M}}=h_{\alpha \beta} d x^{\alpha} d x^{\beta}$, with $\alpha, \beta$ being indices for the time $t$ and the angles $\theta_{i}$, one finds that the determinant of the induced metric is $h=\left(1-\frac{r_{+}^{d-3}}{r^{d-3}}\right) r^{2(d-2)} \prod_{i=1}^{d-2} \sin ^{2(d-2-i)} \theta_{i}$. The extrinsic curvature of the boundary at $r$ in $d$ dimensions can be calculated to be $K=\frac{d-2}{r} \sqrt{1-\frac{r_{d-3}^{d-3}}{r^{d-3}}+}$ $\frac{d-3}{2 r \sqrt{1-\frac{r_{-}^{d-3}}{r^{d-3}}}}\left(\frac{r_{+}}{r}\right)^{d-3}$. The flat counterpart can be obtained by setting $r_{+}=0, K_{\text {flat }}=\frac{d-2}{r}$. To perform the integral in Eq. (11), note that the coordinates on the boundary, $t$ and $\theta_{i}$, can be separated into an integral over the time component and an integral over the angles, so that $I=\frac{[K]}{8 \pi} \sqrt{1-\frac{r_{-}^{d-3}}{r^{d-3}}} \int_{0}^{\beta_{\infty}} d t \int_{\Omega_{d-2}} r^{d-2} d \Omega_{d-2}$, where the Euclidean time is integrated over the period $\beta_{\infty}$ defined above, i.e., $\beta_{\infty}=\frac{4 \pi r_{+}}{d-3}$. Using $[K]=K-K_{\text {flat, }}$, the black hole Euclidean action as a function of the cavity's boundary radius $r$ and the gravitational radius $r_{+}$is then

$$
\begin{aligned}
I= & \frac{(d-1) \Omega_{d-2}}{4(d-3)} r_{+}^{d-2}-\frac{(d-2) \Omega_{d-2}}{2(d-3)} r_{+} r^{d-3} \\
& +\frac{(d-2) \Omega_{d-2}}{2(d-3)} r_{+} r^{d-3} \sqrt{1-\frac{r_{+}^{d-3}}{r^{d-3}}} .
\end{aligned}
$$

In this form, one has that $I=I\left(r, r_{+}\right)$.

Since the thermodynamic variables that fix the canonical ensemble are $r$ and $\beta$, or, equivalently, $r$ and $T$ if one prefers, we want to write the action (12) as a function of $r$ and $\beta$ only, $I=I(r, \beta)$. Noting that $r_{+}=r_{+}(r, \beta)$, see Eqs. (6) and (7) and Fig. 1, one has that Eq. (12) can be formally rewritten as

$$
\begin{aligned}
I(r, \beta)= & \frac{(d-1) \Omega_{d-2}}{4(d-3)}\left[r_{+}(r, \beta)\right]^{d-2} \\
& -\frac{(d-2) \Omega_{d-2}}{2(d-3)} r_{+}(r, \beta) r^{d-3}+\frac{(d-2) \Omega_{d-2}}{8 \pi T} r^{d-3},
\end{aligned}
$$

with the help of Eqs. (2) and (3) for the last term, where $r_{+}$ stands for $r_{+1}$ and $r_{+2}$, With the approximation found in Eq. (6) for $r_{+1}(r, \beta)$, the action for the small black hole is

$I\left(r, r_{+1}(r, \beta)\right)=\frac{\Omega_{d-2} r^{d-2}}{4(d-3)}\left(\frac{d-3}{4 \pi r T}\right)^{d-2}+\mathcal{O}\left(\frac{1}{(\pi r T)^{d-1}}\right)$, which is always positive. With the approximation found in Eq. (7) for $r_{+2}(r, \beta)$, the action for the large black hole is

$$
\begin{aligned}
I\left(r, r_{+2}(r, \beta)\right)= & -\frac{\Omega_{d-2} r^{d-2}}{4}\left(1-\frac{d-2}{2 \pi r T}+\frac{(d-2)(d-3)}{16(\pi r T)^{2}}\right) \\
& +\mathcal{O}\left(\frac{1}{(\pi r T)^{4}}\right),
\end{aligned}
$$

which will be positive for small values of $\pi r T$, provided they still satisfy the condition for existence of equilibrium given in Eq. (5), and will be negative for all the other values of $\pi r T$. From Eq. (12), one can also take that the action of the larger black hole is positive for $\frac{r}{r_{+}}>\left(\frac{(d-1)^{2}}{4(d-2)}\right)^{\frac{1}{d-3}}$ and is negative for $\frac{r}{r_{+}}<\left(\frac{(d-1)^{2}}{4(d-2)}\right)^{\frac{1}{d-3}}$. Since to have a system at all one must impose $r>r_{+}$, the action exists and is negative for $1<\frac{r}{r_{+}}<\left(\frac{(d-1)^{2}}{4(d-2)}\right)^{\frac{1}{d-3}}$, which can only be achieved by the larger black hole $r_{+2}$. Thus, in brief, the action given in Eq. (12) is zero or positive for

$$
\frac{r}{r_{+}} \geq\left(\frac{(d-1)^{2}}{4(d-2)}\right)^{\frac{1}{d-3}}
$$

Note that $\left(\frac{(d-1)^{2}}{4(d-2)}\right)^{\frac{1}{d-3}}$ sets an important cavity radius $r$ in terms of $r_{+}$, the Buchdahl radius, as we will discuss below; see also Appendix B.

\section{THERMODYNAMICS}

The statistical mechanics canonical ensemble setting of black holes is given through the partition function $Z$ and its action $I$ in Eq. (10), where $I$ takes the form of Eq. (12), or Eq. (13), and the connection to thermodynamics is made by the relation between $I$ and the free energy $F$, the relevant thermodynamic potential usually used in the canonical context. The needed relation is

$$
I=\beta F .
$$

In thermodynamics, the thermodynamic energy $E$ and the entropy $S$ are also important thermodynamic potentials, and the relation between $F, E$, and $S$ is

$$
F=E-T S
$$

Now, to establish the first law of thermodynamics, we envisage $E$ as the main thermodynamic potential and assume it to be a function of the entropy $S$ and the cavity area $A, E=E(S, A)$. The first law of thermodynamics can then be written as

$$
d E=T d S-p d A,
$$


where $T$ is the thermodynamic variable conjugated to $S$, i.e., the temperature, that has to be found as an equation of state of the form $T=T(S, A)$, and $p$ is the thermodynamic variable conjugated to $A$, i.e., the tangential pressure or the pressure perpendicular to the cavity radius $r$, that has to be found as an equation of state of the form $p=p(S, A)$. All quantities, $E, T, S, p$, and $A$, are local or quasilocal quantities defined at the cavity's location. To perform calculations directly with the action $I$ given in Eq. (12), or Eq. (13), one changes variables in the first law Eq. (19) to the variable $F$ and then to $I$ using Eq. (18) followed by Eq. (17). We have $d F=d E-T d S-S d T$ and $d I=\beta d F+F d \beta$, so that the first law can be written as

$$
d I=E d \beta-p \beta d A,
$$

i.e., $I=I(\beta, A)$. Then, $E, p$ and $S$ are given by

$$
\begin{gathered}
E=\left(\frac{\partial I}{\partial \beta}\right)_{A}, \\
p=-\frac{1}{\beta}\left(\frac{\partial I}{\partial A}\right)_{\beta} \\
S=\beta E-I,
\end{gathered}
$$

respectively. We can now find $E, p$, and $S$.

To obtain the thermodynamic $E$, we have to perform the derivative $\left(\frac{\partial I}{\partial \beta}\right)_{A}$. It is simpler to use the cavity radius $r$ instead of its area $A$, which can be done through Eq. (9). If $I$ is seen as $I=I(r, \beta)$ then $d I=\left(\frac{\partial I}{\partial \beta}\right)_{r} d \beta+\left(\frac{\partial I}{\partial r}\right)_{\beta} d r$. If $I$ is seen as $I=I\left(r, r_{+}\right)$, then $d I=\left(\frac{\partial I}{\partial r_{+}}\right)_{r} d r_{+}+\left(\frac{\partial I}{\partial r}\right)_{r_{+}} d r$. Equating these two equations at constant $r$, one obtains $\left(\frac{\partial I}{\partial \beta}\right)_{r}=\frac{\left(\partial I / \partial r_{+}\right)_{r}}{\left(\partial \beta / \partial r_{+}\right)_{r}}$. Using Eqs. (2) and (12) in Eq. (21) yields

$$
E=\frac{(d-2) \Omega_{d-2} r^{d-3}}{8 \pi}\left(1-\sqrt{1-\frac{r_{+}^{d-3}}{r^{d-3}}}\right)
$$

The total thermodynamic energy is larger than the spacetime mass $m$, and one can decompose the spacetime mass as the thermodynamic energy inside the cavity minus its gravitational binding energy, i.e., $m=E-\frac{4 \pi E^{2}}{(d-2) \Omega_{d-2} r^{d-3}}$, where $r_{+}^{d-3}=\frac{16 \pi}{(d-2) \Omega_{d-2}} m$ has been used. This thermodynamic energy $E$ is also a quasilocal energy [19].

To obtain the thermodynamic pressure $p$, note that $\left(\frac{\partial I}{\partial r}\right)_{\beta}=\left(\frac{\partial I}{\partial r}\right)_{r_{+}}-\left(\frac{\partial I}{\partial \beta}\right)_{r}\left(\frac{\partial \beta}{\partial r}\right)_{r_{+}}$, where again it is simpler to use the cavity radius $r$ instead of its area $A$, which can be done through Eq. (9). Using Eqs. (2) and (12) in Eq. (22) yields

$$
p=\frac{d-3}{16 \pi r \sqrt{1-\frac{r_{+}^{d-3}}{r^{d-3}}}}\left(1-\sqrt{1-\frac{r_{+}^{d-3}}{r^{d-3}}}\right)^{2}
$$

To obtain the entropy $S$, we use Eqs. (13) and (24) in Eq. (23) to yield

$$
S=\frac{\Omega_{d-2} r_{+}^{d-2}}{4}
$$

This is the Bekenstein-Hawking entropy for black holes in $d$ dimensions.

Having derived the important thermodynamic quantities, we can now find how the number of dimensions $d$ affects the Euler relation and the Gibbs-Duhem relation. From the equations for the thermodynamic energy and entropy, Eqs. (24) and (26), we can write $E=\frac{(d-2) \Omega^{\frac{1}{d-2}}}{8 \pi} A^{\frac{d-3}{d-2}}\left(1-\sqrt{1-\left(\frac{4 S}{A}\right)^{\frac{d-3}{d-2}}}\right)$. So, from Euler's theorem on homogeneous functions, we find that $E$ is homogeneous of degree $\frac{d-3}{d-2}$ in $S$ and $A$, i.e., $\frac{d-3}{d-2} E=\left(\frac{\partial E}{\partial S}\right) S+\left(\frac{\partial E}{\partial A}\right) A$, which means

$$
\frac{d-3}{d-2} E=T S-p A
$$

This is the Euler relation for $d$-dimensional black holes in the canonical ensemble. Taking the differential of the Euler relation in Eq. (27) and using the first law in Eq. (19), we obtain

$$
d E+(d-2) S d T-(d-2) A d p=0,
$$

which is the Gibbs-Duhem relation for $d$-dimensional black holes. In addition, the scaling laws for the gravitational canonical ensemble in $d$ dimensions can be deduced to be $r \rightarrow \lambda r \quad\left(A \rightarrow \lambda^{d-2} A\right), \quad T \rightarrow \lambda^{-1} T(\beta \rightarrow \lambda \beta), E \rightarrow \lambda^{d-3} E$, $S \rightarrow \lambda^{d-2} S$. Curved space is responsible for the fact that intensive parameters lose their homogeneity of degree zero; i.e., the Tolman temperature formula for thermal equilibrium in curved space forces the temperature to lose its usual intensive character. The same happens with the pressure, which now scales as $p \rightarrow \lambda^{-1} p$, a scaling that comes about because it is a pressure that acts in an area $A$ rather than in a volume. Consequently, extensive parameters such as the energy also lose their homogeneity of degree 1 . The action $I$ scales as $I \rightarrow \lambda^{d-2} I$, and the free energy $F$ scales as $F \rightarrow \lambda^{d-3} F$.

\section{THERMAL STABILITY}

The heat capacity at constant cavity area, $C_{A}$, defined by

$$
C_{A} \equiv\left(\frac{\partial E}{\partial T}\right)_{A}
$$


determines the thermal stability of a system in the canonical ensemble. The thermodynamic energy $E\left(r_{+}, r\right)$ is given in Eq. (21), and $r_{+}(\beta, r)$ is given through Eq. (4). Since $T=\frac{1}{\beta}$, see Eq. (3), and since $A=$ const implies $r=$ const, see Eq. (9), one finds that $\left(\frac{\partial E}{\partial T}\right)_{A}=-\beta^{2} \frac{\left(\partial E / \partial r_{+}\right)_{r}}{\left(\partial \beta / \partial r_{+}\right)_{r}}$. Then, the heat capacity for a black hole in $d$ dimensions is given by

$$
C_{A}=\frac{(d-2)}{2(d-1)} \Omega_{d-2} r_{+} r^{d-3} \frac{1-\frac{r_{+}^{d-3}}{r^{d-3}}}{1-\frac{2}{d-1} \frac{r^{d-3}}{r_{+}^{d-3}}} .
$$

A system is thermally stable if

$$
C_{A} \geq 0
$$

Using Eq. (30) on Eq. (31) yields $r_{+} \leq r \leq\left(\frac{d-1}{2}\right)^{\frac{1}{d-3}} r_{+}$. Since $\left(\frac{d-1}{2}\right)^{\frac{1}{d-3}} r_{+}$is the photon orbit radius, $r_{\mathrm{ph}}=\left(\frac{d-1}{2}\right)^{\frac{1}{d-3}} r_{+}$, see also Appendix B, one has

$$
r_{+} \leq r \leq r_{\mathrm{ph}}
$$

i.e., the cavity's boundary $r$ must lie between the black hole and its photon sphere radius (see Fig. 1, and see also Ref. [13]). The smaller black hole $r_{+1}$ given in Eq. (6) will always have its photon sphere inside the cavity radius $r$ and so is thermodynamically unstable. The larger black hole $r_{+2}$ given in Eq. (7) will have its photon sphere outside the cavity radius $r$ and so is thermodynamically stable.

It is interesting to comment on the appearance of the photon orbit radius, $r_{\mathrm{ph}}$, in the context of thermodynamics of black holes, more precisely, in the context of black holes in the canonical ensemble; see also Appendix B. The photon orbit radius appears naturally in the context of particle dynamics in a Schwarzschild background. At this radius, massless particles traveling at the speed of light can have circular orbits. In four dimensions, the photon orbit radius is $r_{\mathrm{ph}}=\frac{3}{2} r_{+}$; in five dimensions, it is $r_{\mathrm{ph}}=\sqrt{2} r_{+}$; and in generic $d$ dimensions, it is $r_{\mathrm{ph}}=\left(\frac{d-1}{2}\right)^{\frac{1}{d-3}} r_{+}$[23]. It is a surprise that the bound also appears in a thermodynamic context. In this context, the bound states that in a canonical ensemble with the boundary radius given by $r$, the black hole is thermodynamically marginally stable if $r_{\mathrm{ph}}=r$, is unstable if $r_{\mathrm{ph}}<r$, and stable if $r_{\mathrm{ph}}>r$. The two contexts, particle dynamics in a Schwarzschild background on one side and black hole thermodynamic stability on the other, are somehow correlated, although this correlation has not been clearly interpreted.

\section{GENERALIZED FREE ENERGY FUNCTION}

Thermodynamics is valid for stationary and thermodynamic stable systems. We have seen that there are two black hole solutions. One, the small black hole solution with horizon radius $r_{+1}$, is unstable, the other, the large black hole solution with horizon radius $r_{+2}$, is stable. So, the whole thermodynamic procedure is valid in principle only for the $r_{+2}$ black hole. For this black hole, there is a welldefined action $I\left(r, r_{+2}\right)$ given in Eq. (15) in an approximation, and its thermodynamic free energy is also well defined since $F\left(r, r_{+2}\right)=\frac{I\left(r, r_{+2}\right)}{\beta}$; see Eq. (17).

We can perturb the free energy $F$ by keeping fixed the quantities that define the canonical ensemble, precisely, the cavity radius $r$ and temperature $T$, and allow $r_{+}$to vary from zero ro $r$. This generalized free energy, $\bar{F}$, is then

$$
\begin{aligned}
\bar{F}\left(r_{+} ; r, T\right)= & \frac{(d-2) \Omega_{d-2} r^{d-3}}{8 \pi} \\
& \times\left(1-\sqrt{1-\frac{r_{+}^{d-3}}{r^{d-3}}}-\frac{2 \pi r T}{(d-2)}\left(\frac{r_{+}}{r}\right)^{d-2}\right),
\end{aligned}
$$

valid for $0 \leq r_{+} \leq r$, and where we have used Eq. (18) together with Eqs. (24) and (26).

The generalized free energy $\bar{F}$ in Eq. (33) has several important properties. For $r_{+}=0$, i.e., the situation where there is no black hole, one has $F=0$. The no black hole situation represents classical hot flat space, i.e., nothing in a Minkowski spacetime, and so it is consistent that it has zero free energy. Also, $\bar{F}$ has two stationary points as one readily finds by computing $\left(\frac{\partial \bar{F}}{\partial r_{+}}\right)_{r, T}=0$. The first stationary point is a local maximum and can be seen to correspond to the small black hole $r_{+1}$, with $\bar{F}\left(r_{+1}\right)$, in thermal equilibrium but unstable; see Eq. (4). The second stationary point is a local minimum and can be seen to correspond to the large black hole $r_{+2}$, with $\bar{F}\left(r_{+1}\right)$, in thermodynamic equilibrium and stable; see Eq. (4). Interpreting $\bar{F}$ as the thermodynamic potential of the ensemble, one can say that the smaller black hole solution $r_{+1}$ acts as a potential barrier separating two stable solutions, classical hot flat space at $r_{+}=0$ with $\bar{F}=0$, and the large black hole $r_{+2}$ with $\bar{F}=\bar{F}\left(r_{+2}\right)$. In general, $\bar{F}\left(r_{+2}\right) \leq \bar{F}\left(r_{+1}\right)$, i.e., $\pi r T \geq \frac{d-3}{4}\left[\left(\frac{2}{d-1}\right)^{\frac{2}{d-3}}-\left(\frac{2}{d-1}\right)^{\frac{d-1}{d-3}}\right]^{-1 / 2}$, the equality holding when $r_{+2}=r_{+1}$.

Moreover, $\bar{F}$ given in Eq. (33) also signals phase transitions. In the canonical ensemble, phase transitions occur always in the direction of decreasing free energy, in this case decreasing $\bar{F}$. One can then study whether there is no possibility of the occurrence of a phase transition from classical hot flat space to the stable black hole $r_{+2}$ or, what here amounts to the same thing, whether there is the possibility that a phase transition from the stable black hole $r_{+2}$ to classical hot flat space can occur, and in which conditions. One can also study, complementarily, whether there is the possibility of the occurrence of a phase transition from classical hot flat space to the stable black hole $r_{+2}$, and in which conditions. Figure 2 gathers all the 

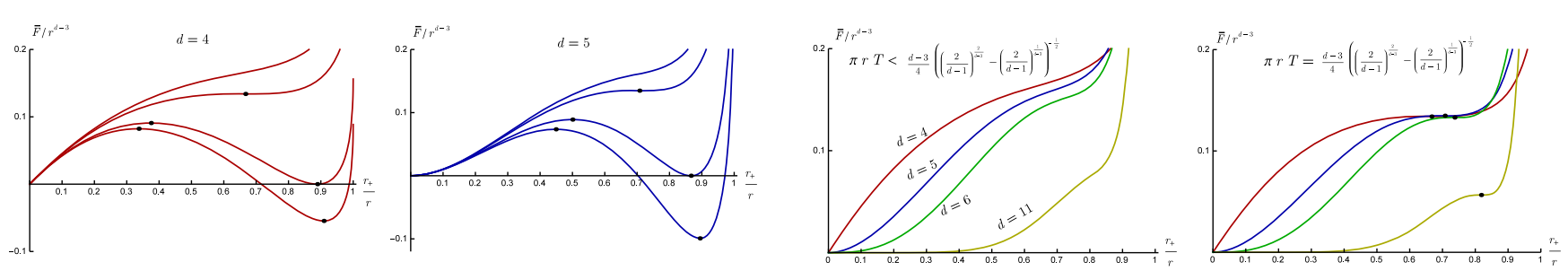

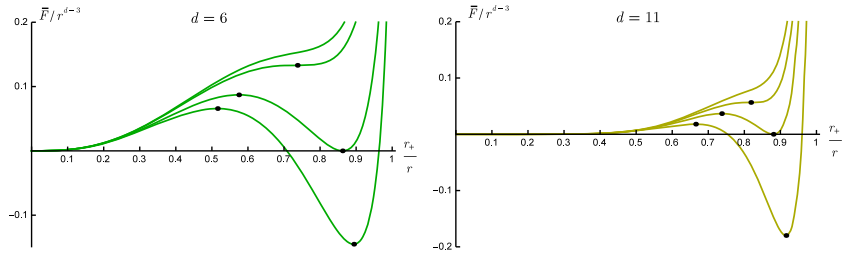

(a)

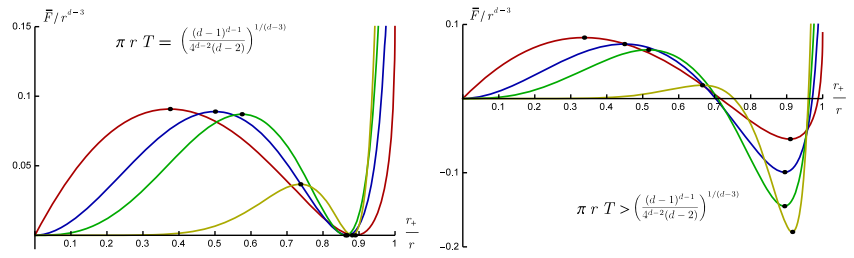

(b)

FIG. 2. Parts (a) and (b) of the figure are complementary; they contain the same information but present it differently. (a) The free energy function $\bar{F}$ is plotted as a function of the horizon radius $r_{+}$for four different dimensions, $d=4,5,6$, and 11 . For each dimension, one has the four typical curves shown according to the value of $\pi r T$. In each plot, the free energy $\bar{F}$ is adimensionalized in terms of the cavity radius $r$ as $\frac{\bar{F}}{r^{d-3}}$, and the horizon radius is also normalized to $r$ as $\frac{r_{+}}{r}$, so that $0 \leq \frac{r_{+}}{r} \leq 1$. The black hole solutions $r_{+1}$ and $r_{+2}$, when they exist, occur at the extrema $\left(\frac{\partial \bar{F}}{\partial r_{+}}\right)_{r, T}=0$; the black dots serve to indicate them. For each dimension, the upper curve is for $\pi r T<\frac{d-3}{4}\left[\left(\frac{2}{d-1}\right)^{\frac{2}{d-3}}-\left(\frac{2}{d-1}\right)^{\frac{d-1}{d-3}}\right]^{-\frac{1}{2}}$, when there are no black hole solutions. For each dimension, the curve below the upper curve is for the limiting situation $\pi r T=\frac{d-3}{4}\left[\left(\frac{2}{d-1}\right)^{\frac{2}{d-3}}-\left(\frac{2}{d-1}\right)^{\frac{d-1}{d-3}}\right]^{-\frac{1}{2}}$, where the two black hole solutions coincide, $r_{+1}=r_{+2}$, in an inflection point, in which situation there is neutral equilibrium. For each dimension, the curve above the lower curve is for $\pi r T=\left(\frac{(d-1)^{d-1}}{4^{d-2}(d-2)}\right)^{\frac{1}{d-3}}$, where the smaller black hole $r_{+1}$ has positive free energy and is unstable, and the larger black hole $r_{+2}$ has zero free energy and is stable. For each dimension, the lower curve is for $\pi r T>\left(\frac{(d-1)^{d-1}}{4^{d-2}(d-2)}\right)^{\frac{1}{d-3}}$, where the smaller black hole $r_{+1}$ has still positive free energy and is unstable, and the larger black hole $r_{+2}$ has now negative free energy and is stable. In the upper two curves, it is not possible for classical hot flat space $r_{+}=0$ which has zero free energy to transition to the large $r_{+2}$ black hole, but the $r_{+2}$ black hole can transition to classical hot flat space. In the lower two curves, classical hot flat space $r_{+}=0$ can nucleate into the large $r_{+2}$ black hole through the small black hole $r_{+1}$. (b) The free energy function $\bar{F}$ is plotted as a function of the horizon radius $r_{+}$ for the four typical situations, namely, $\pi r T<\frac{d-3}{4}\left[\left(\frac{2}{d-1}\right)^{\frac{2}{d-3}}-\left(\frac{2}{d-1}\right)^{\frac{d-1}{d-3}}\right]^{-\frac{1}{2}}, \pi r T=\frac{d-3}{4}\left[\left(\frac{2}{d-1}\right)^{\frac{2}{d-3}}-\left(\frac{2}{d-1}\right)^{\frac{d-1}{d-3}}\right]^{-\frac{1}{2}}, \pi r T=\left(\frac{(d-1)^{d-1}}{4^{d-2}(d-2)}\right)^{\frac{1}{d-3}}$, and $\pi r T>\left(\frac{(d-1)^{d-1}}{4^{d-2}(d-2)}\right)^{\frac{1}{d-3}}$. For each typical situation, one has the four curves corresponding to the four different dimensions, $d=4, d=5$, $d=6$, and $d=11$. In each plot, the free energy $\bar{F}$ is adimensionalized in terms of the cavity radius $r$ as $\frac{\bar{F}}{r^{d-3}}$, and the horizon radius is also normalized to $r$ as $\frac{r_{+}}{r}$, so that $0 \leq \frac{r_{+}}{r} \leq 1$.

necessary information to study these phase transitions by plotting the free energy function $\bar{F}$ as a function of the horizon radius in units of the cavity radius, $\frac{r_{+}}{r}$, as given in Eq. (33), for four different dimensions, $d=4, d=5$, $d=6$, and $d=11$, and for each dimension, giving the four important different situations that depend on the value of $\pi r T$, and, to complement, by also plotting the free energy function $\bar{F}$ as a function of the horizon radius in units of the cavity radius, $\frac{r_{+}}{r}$, as given in Eq. (33), for the four important different situations that depend on the value of $\pi r T$, and in each situation showing the four different dimensions, $d=4, d=5, d=6$, and $d=11$. Let us see in detail these phase transitions. We start the analysis in the case that there is no possibility of the occurrence of a phase transition from classical hot flat space to the stable black hole $r_{+2}$. Or, what here amounts to the same thing, in the case that a phase transition from the stable black hole $r_{+2}$ to classical hot flat space can occur. Since classical hot flat space has zero free energy $\bar{F}=0$, one has that a phase transition from the stable black hole $r_{+2}$ to classical hot flat space can occur when $\bar{F}\left(r_{+2}\right) \geq 0$, i.e., $I\left(r_{+2}\right) \geq 0$. By repeating the analysis done from Eqs. (12)-(16), one can find that $I\left(r_{+2}\right) \geq 0$ when $\frac{r_{+}}{r} \leq \frac{r_{+}}{r_{\mathrm{Buch}}}$. where $r_{\mathrm{Buch}}$ is the $d$-dimensional Buchdahl radius given by $r_{\text {Buch }}=$ $\left(\frac{(d-1)^{2}}{4(d-2)}\right)^{\frac{1}{d-3}} r_{+}$. Together with the condition for the existence of black holes in thermodynamic equilibrium, i.e., Eq. (5), one finds that a large black hole $r_{+2}$ can decay into classical hot flat space when

$$
\begin{aligned}
& \frac{d-3}{4}\left[\left(\frac{2}{d-1}\right)^{\frac{2}{d-3}}-\left(\frac{2}{d-1}\right)^{\frac{d-1}{d-3}}\right]^{-\frac{1}{2}} \\
& \quad \leq \pi r T \leq\left(\frac{(d-1)^{d-1}}{4^{d-2}(d-2)}\right)^{\frac{1}{d-3}}, \quad r_{\text {Buch }} \leq r<\infty
\end{aligned}
$$


Also, when $\pi r T$ and $r$ obey Eq. (34), classical hot flat space never nucleates into a black hole. We now analyze the inverse transition, i.e., the transition from classical hot flat space to the stable black hole. Since classical hot flat space has zero free energy $\bar{F}=0$, one has that a phase transition to the stable black hole $r_{+2}$ can occur when $\bar{F}\left(r_{+2}\right) \leq 0$, i.e., $I\left(r_{+2}\right) \leq 0$. From Eq. (12), we have done the analysis ending in Eq. (16); i.e., we have found that $I\left(r_{+2}\right) \leq 0$ when $\frac{r_{+}}{r} \geq \frac{r_{+}}{r_{\text {Buch }}}$ where $r_{\text {Buch }}$ is the $d$-dimensional Buchdahl radius given by $r_{\mathrm{Buch}}=\left(\frac{(d-1)^{2}}{4(d-2)}\right)^{\frac{1}{d-3}} r_{+}$. Putting this back into Eq. (33), one can see that this happens for $\pi r T \geq\left(\frac{(d-1)^{d-1}}{4^{d-2}(d-2)}\right)^{\frac{1}{d-3}}$. Thus, a transition from classical hot flat space to the stable black hole occurs for

$$
\left(\frac{(d-1)^{d-1}}{4^{d-2}(d-2)}\right)^{\frac{1}{d-3}} \leq \pi r T \leq \infty, \quad r_{+} \leq r \leq r_{\mathrm{Buch}} .
$$

Equation (35) is a necessary and sufficient condition for the occurrence of nucleation from classical hot flat space to the stable black hole $r_{+2}$, a transition that is done through the unstable black hole $r_{+1}$. We also see that Eq. (35) imposes a stronger condition than the $\pi r T \geq$ $\frac{d-3}{4}\left[\left(\frac{2}{d-1}\right)^{\frac{2}{d-3}}-\left(\frac{2}{d-1}\right)^{\frac{d-1}{d-3}}\right]^{-\frac{1}{2}}$ of Eq. (5) for having black holes in thermodynamic equilibrium at all.

It is interesting to comment on the appearance of the Buchdahl radius, $r_{\text {Buch }}$, in the context of thermodynamics of black holes, more precisely, in the context of black holes in the canonical ensemble; see also Appendix B. The Buchdahl bound has appeared in the context of general relativistic star structure. It is a bound that states that under some generic conditions for a star of radius $r$, the spacetime is free of singularities for $r_{\text {Buch }} \leq r$. It is a lower bound for the ratio $\frac{r}{r_{+}}$, where $r$ is the star's radius and $r_{+}$its gravitational radius, that appears such that the star spacetime is singularity free. Presumably, for $r_{\text {Buch }} \geq r$, the star might collapse into a black hole. In four dimensions, the limiting radius of the bound is $r_{\mathrm{Buch}}=\frac{9}{8} r_{+}$; in five dimensions, it is $r_{\mathrm{Buch}}=\frac{2}{\sqrt{3}} r_{+}$; and in generic $d$ dimensions, the limiting radius of the bound is $r_{\text {Buch }}=$ $\left(\frac{(d-1)^{2}}{4(d-2)}\right)^{\frac{1}{d-3}} r_{+}$(see Refs. [20,21] for four dimensions and Ref. [24] for $d$ dimensions). It is a surprise that the bound also appears in a thermodynamic context. In this context, the bound we have found states that in a canonical ensemble with the boundary radius given by $r$, classical hot flat space cannot transition to a black hole phase if $r_{\text {Buch }} \leq r$. If, contrarily, $r_{\text {Buch }} \geq r$, then classical hot flat space can make a transition to a black hole. The two contexts, general relativistic star solutions and gravitational collapse on one side and black hole thermodynamic on the other, are thus clearly correlated, and thus this correlation hints that $r_{\text {Buch }}$ is an intrinsic property of the Schwarzschild spacetime, as the radius of the photon orbit, $r_{\mathrm{ph}}$, is. To corroborate this statement and explicitly see this correlation, a comparison of the thermodynamics of Schwarzschild black holes and classical hot flat space in a cavity with radius $r$ at a fixed temperature $T$ in the canonical ensemble in $d$ dimensions with the thermodynamics of a self-gravitating thin shell of radius $r$ and at temperature $T$ with a Minkowski interior and a Schwarzschild exterior can be performed; see Appendix C.

\section{ACTION FUNCTIONAL TO SECOND ORDER AND ITS ROLE IN THERMODYNAMIC STABILITY}

The path integral approach to a quantum gravity system prescribes that one must integrate the exponential of the negative of the Euclidean Einstein action $I$ over the space of metrics $g$ to obtain the canonical partition function of the system, $Z=\int d[\mathrm{~g}] \exp (-I[\mathrm{~g}])$. For a black hole system with classical action $I$, one can use the zeroth order approximation yielding $Z=\exp (-I)$; see Eq. (10). One can go a step further and perturb the Euclidean black hole metric $\bar{g}_{a b}$ by a small amount $h_{a b}$, such that the full perturbed metric is $g_{a b}=\bar{g}_{a b}+h_{a b}$, where clearly $\bar{g}_{a b}$ is envisaged now as a background solution and $h_{a b}$ is envisaged as a small fluctuation. The Euclidean action can then be approximated by $I[\mathrm{~g}]=I[\overline{\mathrm{g}}]+$ $\int d^{4} x \sqrt{\overline{\mathrm{g}}} A_{a b c d} h^{a b} h^{c d}$, for some operator $A_{a b c d}$ which generically depends on the metric $\bar{g}_{a b}$, its covariant derivatives, and curvature terms. There are two possibilities depending on the perturbation operator $A_{a b c d}$. If one of the eigenvalues of $A_{a b c d}$ is negative, then the integral gets an imaginary term, which implies that the action and the free energy have an imaginary term and the partition function will also contain an imaginary part. In this case, the original classical black hole instanton is a saddle point, and it is unstable. On the contrary, there is the possibility that all of the eigenvalues of $A_{a b c d}$ are positive, in which case the perturbation modes are stable around the given black hole solution.

In four dimensions, the perturbation performed around the Euclidean Schwarzschild black hole solution with a cavity with a very large radius $r$ at a fixed temperature $T$ yielded that the operator $A_{a b c d}$ has indeed a negative eigenvalue, resulting in an instability [6]. Connecting this result to thermodynamic stability, it means that a black hole in thermodynamic equilibrium in the canonical ensemble with a large cavity cannot be thermodynamically stable. However, when the cavity radius $r$ is reduced, one finds [7] that the negative mode vanishes below a certain radius $r=\frac{3}{2} r_{+}$of the cavity, indicating stability. Connecting the result to thermodynamic stability, it means that a black hole in thermodynamic equilibrium in the canonical ensemble can be thermodynamically stable for $r \leq \frac{3}{2} r_{+}$. This correspondence between perturbation path integral 
theory and thermodynamic stability was found by York [5], establishing that there are actually two Euclidean solutions in thermal equilibrium, one of which is in an unstable thermodynamic equilibrium which has negative heat capacity, the smaller one denoted by $r_{+1}$, and one which is in stable thermodynamic equilibrium which has positive heat capacity, the larger one denoted by $r_{+2}$. The condition for stable thermodynamic equilibrium matched exactly the condition for stability of the solution.

In $d$ dimensions, one can also work out a perturbation analysis on the path integral [13] to find that $d$-dimensional Schwarzschild black holes have a negative mode if the black hole radius $r_{+}$is small compared to the cavity radius $r$, i.e., there is a negative mode for the $r_{+1}$ black hole, and have no negative mode if the black hole radius $r_{+}$is of the order of the cavity radius $r$, i.e., there is no negative mode for the $r_{+2}$ black hole, the marginal zero mode case being when the cavity radius $r$ is at the photon orbit radius $r=r_{\mathrm{ph}}$. These results are thus also in one-to-one correspondence with the instability or stability thermodynamic analysis done through the heat capacity of the $d$-dimensional black hole. In Ref. [14], it was further clarified that thermodynamic stability of black holes and the mechanic stability of black systems, such as black branes, are interrelated.

\section{GROUND STATE OF THE CANONICAL ENSEMBLE: QUANTUM HOT FLAT SPACE, BLACK HOLE, OR BOTH}

From the partition function of black holes and the thermodynamic stability, as well as from the perturbation studies on the action functional, it is clear that in order to properly understand the physics involved, one has to treat hot flat space in quantum terms; i.e., hot flat space should be treated as made of hot gravitons. In this way, the issues of what is the ground state of the canonical ensemble and what are the possible phase transitions can be addressed.

In the canonical ensemble, the ground state is the one that has the lowest free energy $F$ or, if one prefers, the lowest action $I$, as $I=\beta F$. For the hot gravity system under study, the three possible phases are quantum hot flat space, the phase of a stable black hole with large radius $r_{+2}$, or a possible superposition of these two phases. The black hole with small radius $r_{+1}$ is not a phase since it is unstable, as found previously. Thus, to find the ground state of hot gravity in the canonical ensemble, i.e., hot gravity at a given temperature $T$ and a given cavity radius $r$, the free energy of quantum hot flat space $F_{\mathrm{HFS}}$ and the free energy of the large black hole $F\left(r_{+2}\right)$ must be compared.

Minkowski flat space has $r_{+}=0$ and in the context of hot gravity is also a solution in thermal equilibrium; i.e., fixing the temperature at the cavity boundary, the temperature will be the same everywhere inside the cavity. As follows from the Stefan-Boltzmann law, quantum hot flat space, or flat space at finite temperature, has finite free energy and thus finite action. For a $d$-dimensional system containing only gravitons, which is the case we consider here, the number of massless species is given by $\frac{1}{2} d(d-3)$, and in this case, one finds that the free energy in $d$ dimensions of quantum hot flat space is given by

$$
F_{\mathrm{HFS}}=-\frac{d(d-3)}{2(d-1)^{2}} a \Omega_{d-2} r^{d-1} T^{d}
$$

where $a=\frac{\Gamma(d) \zeta(d)}{2^{d-2} \pi^{\frac{d-1}{2}} \Gamma\left(\frac{d-1}{2}\right)}$, with $\Gamma$ and $\zeta$ being the gamma and zeta functions, respectively; see Appendix D. The free energy of quantum hot flat space is negative, and not zero as in the case of classical hot flat space. Its dependence with the cavity radius $r$ and temperature $T$ is $r^{d-1} T^{d}$. If one prefers to use the action $I$, then since $I=\beta F$ and $\beta=\frac{1}{T}$, one has $I_{\mathrm{HFS}}=-\frac{d(d-3)}{2(d-1)^{2}} a \Omega_{d-2} r^{d-1} T^{d-1}$.

The free energy of the stable black hole $r_{+2}$ is $F\left(r_{+2}\right)$. This free energy can be found using the larger $r_{+2}$ solution of Eq. (4) in Eq. (13) for $I$ and then using $F\left(r_{+2}\right)=T I\left(r_{+2}\right)$. For $d=5$, one can find an exact solution [12], but for any other $d$, either there is no exact solution or, if there is, it is unusable. We can then either resort to the large $T$ approximation for $r_{+2}$ given in Eq. (7) and for $I\left(r_{+2}\right)$ and so $F\left(r_{+2}\right)$ given in Eq. (15) or to numeric calculations. Let us start with the large $T$ approximation. Using Eq. (15) and $F\left(r_{+2}\right)=T I\left(r_{+2}\right)$ yields

$$
\begin{aligned}
F\left(r_{+2}\right)= & -\frac{\Omega_{d-2} r^{d-2} T}{4}\left(1-\frac{d-2}{2 \pi r T}+\frac{(d-2)(d-3)}{16(\pi r T)^{2}}\right) \\
& +\mathcal{O}\left(\frac{d^{4}}{(\pi r T)^{4}}\right)
\end{aligned}
$$

where we have been shortening the notation $F\left(r_{+2}\right) \equiv$ $F\left(r, r_{+2}(r, \beta)\right)$. One could make a plot through numerical calculations of $F\left(r_{+2}\right)$ as a function of $\pi r T$, but it is not so useful.

One has now to compare $F_{\mathrm{HFS}}$ of Eq. (36) with $F\left(r_{+2}\right)$ of Eq. (37) or $F\left(r_{+2}\right)$ given by numerical calculations. The stable black hole $r_{+2}$ is the ground state when

$$
F\left(r_{+2}\right) \leq F_{\mathrm{HFS}}
$$

In the situation that the equality holds, then the black hole and quantum hot flat space phases coexist. For the phase diagram of the gravitational canonical ensemble with a plot of the cavity radius $r$ versus the temperature $T$ for several different dimensions, specifically, $d=4,5,6$, and 11 , see Fig. 3.

Let us first use the approximation given by Eq. (37). Putting Eqs. (36) and (37) into Eq. (38) yields 


$$
\begin{aligned}
r^{-(d-2)} \leq & \frac{\pi^{d-1}(d-1)^{2}}{2 a d(d-3)(\pi r T)^{d-1}} \\
& \times\left(1-\frac{d-2}{2 \pi r T}+\frac{(d-2)(d-3)}{16(\pi r T)^{2}}\right),
\end{aligned}
$$

up to $\mathcal{O}\left(\frac{1}{(r T)^{4}}\right)$. Note that the right-hand side of Eq. (39) has an extremum at $\pi r T=\frac{d(d-2)+\sqrt{(d-2)\left(d^{2}+d-3\right)}}{4(d-1)}$, leading to a minimum radius $r_{\min }$ given by $r_{\min }=$ $\left(\frac{a d(d-3)}{2 \pi^{d-1}}\right)^{\frac{1}{d-2}} \times\left(\frac{\left(\frac{\left.d(d-2)+\sqrt{(d-2)\left(d^{2}+d-3\right.}\right)}{d+1}\right)^{d+1}}{2^{2 d-3}(d-2)\left(2 d-3+\sqrt{(d-2)\left(d^{2}+d-3\right)}\right.}\right)^{\frac{1}{d-2}}, \quad$ below which the black hole will never be the ground state of the ensemble. In more detail, in the case the radius of the cavity is smaller than $r_{\min }$, one has that quantum hot flat space is always the ground state. Therefore, a necessary but not sufficient condition for black hole nucleation from quantum hot flat space is that the radius of the cavity be greater than $r_{\min }$. One finds in these approximations that $r_{\min } \simeq 0.2525$ in $d=4, r_{\min } \simeq 0.4971$ in $d=5, r_{\text {min }} \simeq$ 0.7012 in $d=6$, and $r_{\min } \simeq 1.5636$ in $d=11$. In the large $d$ limit, one has $r_{\min } \rightarrow a^{\frac{1}{d}}$, and since $a=\frac{\Gamma(d) \xi(d)}{2^{d-2} \pi^{\frac{d-1}{2}} \Gamma\left(\frac{d-1}{2}\right)} \rightarrow \infty$, in the $d \rightarrow \infty$ limit, one has that $r_{\min }$ tends to infinity. For $d$ finite, say $d=11, r_{\min } \simeq 1.5636$, i.e., $r_{\min }$ is still near the Planck length which we have set to 1 , but it increases for larger $d$. If one uses numerical calculations, see Fig. 3, then one finds $r_{\min } \simeq 0.2511$ in $d=4, r_{\min } \simeq 0.4915$ in $d=5$, $r_{\text {min }} \simeq 0.6901$ in $d=6$, and $r_{\text {min }} \simeq 1.5187$ in $d=11$. The approximation is in any case excellent.

The inequality of Eq. (38) can also be written in terms of the temperature of the cavity $T$. Let us use the approximation. Then, indeed, Eq. (38) yields

$T^{d-2} \leq \frac{\pi(d-1)^{2}}{2 a d(d-3) \pi r T}\left(1-\frac{d-2}{2 \pi r T}+\frac{(d-2)(d-3)}{16(\pi r T)^{2}}\right)$,

up to $\mathcal{O}\left(\frac{1}{(r T)^{4}}\right)$. Now, the right-hand side has an extremum at $\pi r T=\frac{d-2}{2}+\frac{\sqrt{(d-2)(d+1)}}{4}$, which then leads to finding a maximum value for the temperature $T_{\max }$ given by $T_{\max }=\left(\frac{4 \pi}{a d(d-3)}\right)^{\frac{1}{d-2}} \times\left(\frac{(d-2)(d-1)^{2}(d-1+\sqrt{(d-2)(d+1)})}{(2(d-2)+\sqrt{(d-2)(d+1)})^{3}}\right)^{\frac{1}{d-2}}$, above which the black hole will never be the ground state. In more detail, in the case the temperature is larger than $T_{\max }$, one has that quantum hot flat space is always the ground state. Therefore, a necessary but not sufficient condition for black hole nucleation from quantum hot flat space is that the temperature of the cavity be smaller than $T_{\max }$. One finds in these approximations that $T_{\max } \simeq 1.6979$ in $d=4$, $T_{\max } \simeq 1.1365$ in $d=5, T_{\max } \simeq 0.9827$ in $d=6$, and $T_{\max } \simeq 0.7891$ in $d=11$. In the large $d$ limit, one finds that $T_{\max }$ tends to $T_{\max } \rightarrow \frac{1}{a^{\frac{1}{d}}}$, which, taking into account the
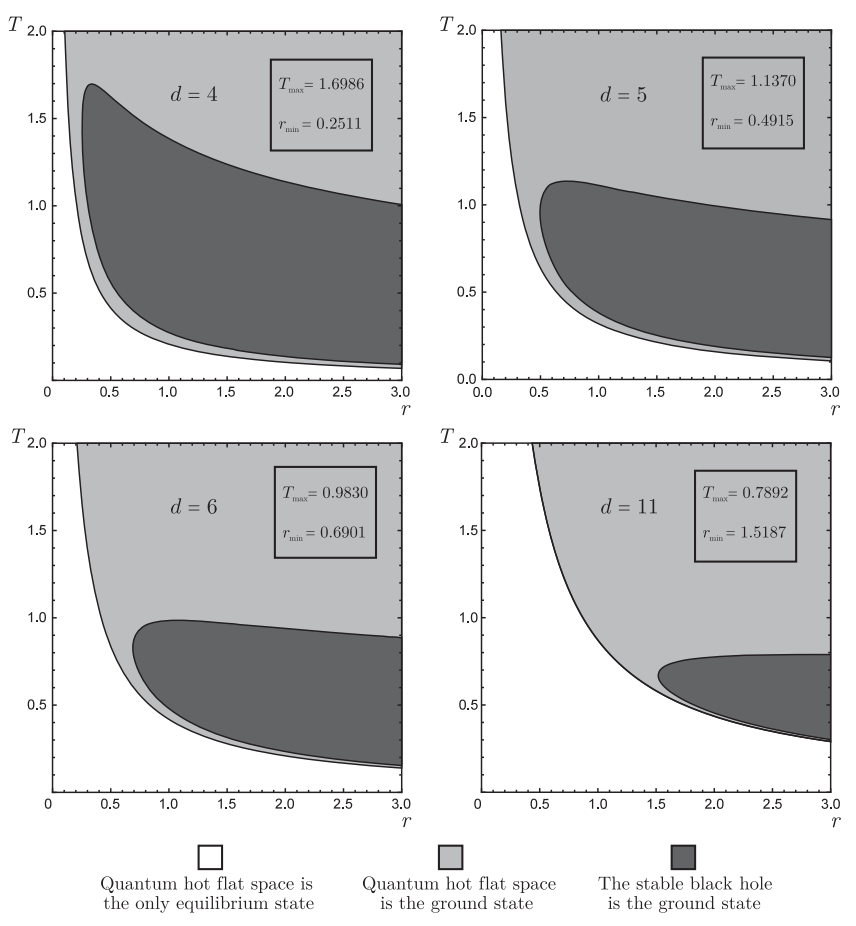

FIG. 3. Phase diagram of the gravitational canonical ensemble with a plot of the cavity radius $r$ versus the temperature $T$, both in Planck units, for four different dimensions, $d=4,5,6$, and 11 . Each point in the plots represents a different configuration of the ensemble. Each plot is separated in three different regions that yield three different phases. The white region in each plot, for which $\pi r T \geq \frac{d-3}{4}\left[\left(\frac{2}{d-1}\right)^{\frac{2}{d-3}}-\left(\frac{2}{d-1}\right)^{\frac{d-1}{d-3}}\right]^{-1 / 2}$ holds, see Eq. (5), is the region, or phase, where there quantum hot flat space is the only equilibrium state; in this region, there are no black holes in thermodynamic equilibrium at all. The gray region in each plot characterizes the phase that has quantum hot flat space as the ground state; i.e., the action for quantum hot flat space is lower than the stable black hole's action [see Eq. (38) with the inequality reversed[. The dark region in each plot characterizes the phase that has the larger stable black hole $r_{+2}$ as the ground state of the canonical ensemble; see Eq. (38) with the inequality holding. A quantum hot flat space configuration in this phase is then able to nucleate stable black holes. The thick black line in each plot yields a mixed phase, i.e., a superposition of the quantum hot flat ground state phase with the stable black hole ground state phase; see Eq. (38) with the equality holding. The extremal values for the radius, $r_{\min }$, and for the temperature, $T_{\max }$, are the lower bound and the upper bound of the dark region, respectively. If instead of quantum hot flat space one were to consider classical hot flat space, i.e., the zero free energy of Minkowski spacetime, the gray and the dark regions would be separated by an asymptote following Eq. (35), which in the classical limit, i.e., $r \gg 1$ and $T \ll 1$, in Planck units, matches the line separating both regions; see Appendix E for further details on classical hot flat space with the corresponding black hole phase transitions.

expression for $a, a=\frac{\Gamma(d) \zeta(d)}{2^{d-2} \pi^{\frac{d-1}{2}} \Gamma\left(\frac{d-1}{2}\right)}$, given the $d \rightarrow \infty$ limit of $a$, tends to 0 . For not so large values of $d$, the maximum temperature does not deviate much from the Planck 
temperature which we set to 1 . If one uses numerical calculations, see Fig. 3, then one finds $T_{\max } \simeq 1.6986$ in $d=4, T_{\max } \simeq 1.1370$ in $d=5, T_{\max } \simeq 0.9830$ in $d=6$, and $T_{\max } \simeq 0.7892$ in $d=11$. One sees that the approximations for the maximum temperature hold better than for the minimum radius for large $d$. The fact that the approximation for $T_{\max }$ holds better than the one for $r_{\min }$ for higher dimensions is because the initial approximation taken, i.e., the solution $r_{+2}$ in Eq. (7), depends on $\pi r T$. From Eq. (39), it was seen that, for large $d, r_{\min }$ will lie on a curve approaching $\pi r T=\frac{d}{4}$, whereas from Eq. (40), for large $d, T_{\max }$ will lie on a curve approaching $\pi r T=\frac{3 d}{4}$, so $T_{\max }$ will be more accurate than $r_{\min }$, since Eq. (7) holds better for larger values of $\pi r T$.

Thus, from Fig. 3, we see that there are three phases. One phase is when the cavity's radius $r$ and the cavity's temperature $T$ are such that in thermodynamic equilibrium only quantum hot flat space is possible, there are no stable equilibrium black holes $r_{+2}$, and for that matter there are also no unstable equilibrium black holes $r_{+1}$, but black holes out of thermodynamic equilibrium may perhaps appear in this phase. Another phase is when the cavity's radius $r$ and the cavity's temperature $T$ are such that quantum hot flat space is the ground state, and so stable black holes $r_{+2}$ can transition into quantum hot flat space. And yet another phase is when the cavity's radius $r$ and the cavity's temperature $T$ are such that the stable black hole $r_{+2}$ is the ground state, and so quantum hot flat space can nucleate stable black holes. These three phases are represented by the white, gray, and dark regions, respectively, in Fig. 3. There is a mixed phase which is a superposition of the quantum hot flat ground state phase with the stable black hole $r_{+2}$ ground state phase, which is represented by a line between the gray and dark regions. A feature that Fig. 3 makes clear is that as the number of spacetime dimensions increases, the region for the quantum hot flat ground state phase gets larger, whereas the region for the stable black hole ground state phase gets smaller. In the $d \rightarrow \infty$ limit, black holes never nucleate as expected.

It is also of interest to understand the passage from quantum hot flat space and black hole phase transitions to classical hot flat space and the corresponding black hole phase transitions. In this passage, one puts the constant $a$ that appears in Eq. (36) to zero, $a=0$, and the analysis follows; see Appendix E.

\section{DENSITY OF STATES}

It is interesting to find through the density of states $\nu$ with a given energy $E$ that the entropy of the $r_{+2}$ black hole is $S=\frac{A_{+2}}{4}$.

Fixing the cavity radius $r$, the number of states between $E$ and $E+d E$ is given by $\nu(E) d E$ with $\nu(E)$ being the density of states. Thus, weighing this density $\nu(E)$ with the Boltzmann factor $e^{-\beta E}$, the canonical partition function can be written as $Z(\beta, r)=\int d E \nu(E) e^{-\beta E}$. Inverting this expression by an inverse Laplace transform, one obtains $\nu(E)=\frac{1}{2 \pi i} \int_{-i \infty}^{i \infty} d \beta Z(\beta) e^{\beta E}$. The partition function for the stable black hole is $Z=\exp \left(-I\left(r, r_{+2}(r, \beta)\right)\right)$. Using $I\left(r, r_{+2}(r, \beta)\right)$ given in Eq. (15) for large $r T$, one finds

$$
\begin{aligned}
Z(\beta, r) \simeq & \exp \left(\frac{\Omega_{d-2} r^{d-2}}{4}-\frac{(d-2) \Omega_{d-2} r^{d-3}}{8 \pi} \beta\right. \\
& \left.+\frac{(d-2)(d-3) \Omega_{d-2} r^{d-4}}{64 \pi^{2}} \beta^{2}\right) .
\end{aligned}
$$

Taking the inverse Laplace transform, one has $\nu(E)=\frac{4 \sqrt{\pi}}{\sqrt{(d-2)(d-3) \Omega_{d-2} r^{d-4}}} \exp \left(\frac{\Omega_{d-2} r^{d-2}}{4}-\frac{16 \pi^{2}}{(d-2)(d-3) \Omega_{d-2} r^{d-4}}\right.$ $\left.\left(E-\frac{(d-2) \Omega_{d-2} r^{d-3}}{8 \pi}\right)^{2}\right)$. Now, the spacetime mass $m$ is given in terms of $E$ and the cavity radius $r$ by $m=E-\frac{4 \pi E^{2}}{(d-2) \Omega_{d-2} r^{d-3}}$. We can then write $\nu(E)$ as $\nu(E)=\frac{4 \sqrt{\pi}}{\sqrt{(d-2)(d-3) \Omega_{d-2} r^{d-4}}} \exp \left(-\frac{\Omega_{d-2} r^{d-2}}{4(d-3)}+\frac{4 \pi r m}{(d-3)}\right)$. Finally, with the equation $r_{+}^{d-3}=\frac{16 \pi}{(d-2) \Omega_{d-2}} m$ along with the fact that $r_{+2} \simeq r$ for the stable black hole in this regime, one finds that the density of states is well described by

$$
\nu(E)=\frac{4 \sqrt{\pi}}{\sqrt{(d-2)(d-3) \Omega_{d-2} r^{d-4}}} \exp \left(\frac{A_{+2}}{4}\right)
$$

where the area of the black hole is $A_{+2}=\Omega_{d-2} r_{+2}^{d-2}$. The entropy $S$ and the density of states $\nu$ are related through the formula $S=a \ln \nu$, for some constant $a$, so the black hole entropy is

$$
S=\frac{A_{+2}}{4},
$$

where we discarded the remaining constant. In contrast, for the unstable black hole $r_{+1}$, the action of Eq. (14) has a divergent integral when one performs the Laplace transform. Only the large stable black hole $r_{+2}$ yields the correct result.

For a synopsis of all the results and further comments, see Appendix F.

\section{ACKNOWLEDGMENTS}

R. A. acknowledges support from the Doctoral Programme in the Physics and Mathematics of Information (DP-PMI) and the Fundação para a Ciência e Tecnologia (FCT) through Grant No. PD/BD/135011/ 2017. J. P. S. L. acknowledges FCT for financial support through Project No. UIDB/00099/2020. 


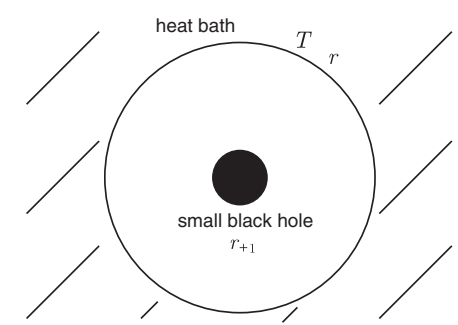

FIG. 4. The small black hole solution, $r_{+1}$ is depicted inside the heat bath cavity characterized by its radius $r$ and temperature $T$.

\section{APPENDIX A: CALCULATION OF THE APPROXIMATE EXPRESSIONS FOR THE CANONICAL ENSEMBLE HORIZON RADII $r_{+1}$ AND $r_{+2}$}

Here, we perform the calculation that lead to the approximate expressions for $r_{+1}$ and $r_{+2}$ from Eq. (4) to Eqs. (6) and (7), respectively. For the sake of quick reference, we repeat Eq. (4), which is

$$
\left(\frac{r_{+}}{r}\right)^{d-1}-\left(\frac{r_{+}}{r}\right)^{2}+\left(\frac{d-3}{4 \pi r T}\right)^{2}=0 .
$$

It is a polynomial equation of order $d-1$, which has direct exact solutions for $d=4$ and $d=5$, whereas for other $d$, one is compelled to resort to approximation schemes or numerical calculations. We display an approximation scheme to find $r_{+1}$ and $r_{+2}$.

For the smaller black hole $r_{+1}$, see Fig. 4 let us write the general form of the solution as a Taylor expansion on $\pi r T$ around $r_{+}=0$. Let us call $r_{+1}$ the gravitational radius of the smaller black hole. Here, we write $r_{+1}=r_{+1}(\pi r T)$ as

$$
r_{+1}=r\left(\sum_{i=1}^{\infty} \frac{a_{i}}{(\pi r T)^{i}}\right)
$$

where the $a_{i}$ are constants to be determined. Now, we need the expanding expressions for $\left(\frac{r_{+1}}{r}\right)^{d-1}$ and $\left(\frac{r_{+1}}{r}\right)^{2}$ so that each power in $\pi r T$ cancels out in Eq. (A1). Using Eq. (A2), we find $\left(\frac{r_{+1}}{r}\right)^{d-1}=\frac{a_{1}^{d-1}}{(\pi r T)^{d-1}}+\frac{(d-1) a_{1}^{d-2} a_{2}}{(\pi r T)^{d}}+\cdots+\frac{2 a_{1} a_{d-2}}{(\pi r T)^{d-1}}+\ldots$ and $\left(\frac{r_{+1}}{r}\right)^{2}=\frac{a_{1}^{2}}{(\pi r T)^{2}}+\frac{2 a_{1} a_{2}}{(\pi r T)^{3}}+\ldots$. Due to the presence of the term $\left(\frac{d-3}{4 \pi r T}\right)^{2}$ in Eq. (A1), $a_{1}$ can never be 0 , and since $d \geq 4$, the lowest possible order in the $\left(\frac{r_{+1}}{r}\right)^{d-1}$ expansion is in $\frac{1}{(\pi r T)^{3}}$, so, the only way for the first term in the $\left(\frac{r_{+1}}{r}\right)^{2}$ expansion to cancel out in Eq. (A1) is by setting $a_{1}=\frac{d-3}{4}$. Moreover, since the lowest order showing up in the $\left(\frac{r_{+1}}{r}\right)^{d-1}$ expansion is in $\frac{1}{(\pi r T)^{d-1}}$, then all orders between -1 and $-(d-2)$ must cancel out by setting the respective coefficient to zero, i.e., $a_{i}=0$ for $2 \leq i \leq d-3$. Thus, the next order term in the expansion is that of $a_{d-2}$. This is because by taking into account that $a_{i}=0$ for $2 \leq i \leq d-3$, the

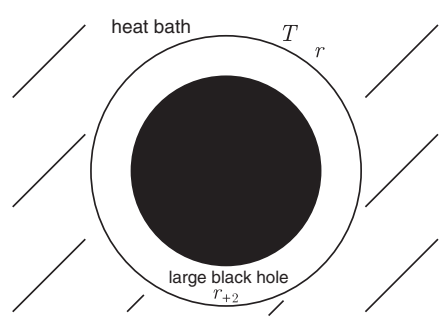

FIG. 5. The large black hole solution $r_{+2}$ is depicted inside the heat bath cavity characterized by its radius $r$ and temperature $T$.

term in $\frac{1}{(\pi r T)^{d-1}}$ arising from the squared term $\left(\frac{r_{+}}{r}\right)^{2}$ in Eq. (A1) is $\frac{2 a_{1} a_{d-2}}{(\pi r T)^{d-1}}$. This term, along with the one coming from the term in the $\left(\frac{r_{+1}}{r}\right)^{d-1}$ expansion, will have to cancel out in Eq. (A1) by setting $a_{d-2}=\frac{1}{2}\left(\frac{d-3}{4}\right)^{d-2}$. With these values for the $a_{i}$, Eq. (A2) is now

$r_{+1}=r\left(\frac{d-3}{4 \pi r T}+\frac{1}{2}\left(\frac{d-3}{4 \pi r T}\right)^{d-2}+\mathcal{O}\left(\frac{1}{(\pi r T)^{d-1}}\right)\right)$.

For the larger black hole $r_{+2}$, see Fig. 5, let us write the general form of the solution as a Taylor expansion on $\pi r T$ around $r$. Let us call $r_{+2}$ the gravitational radius of the larger black hole. Here, we write $r_{+2}=r_{+2}(\pi r T)$ as

$$
r_{+2}=r\left(\sum_{i=0}^{\infty} \frac{b_{i}}{(\pi r T)^{i}}\right)
$$

where the $b_{i}$ for $i \geq 0$ are constants to be determined. Since for $r_{+2}$ the expansion is around $r$, one has $b_{0}=1$. Now, we need the expanding expressions for $\left(\frac{r_{+2}}{r}\right)^{d-1}$ and $\left(\frac{r_{+2}}{r}\right)^{2}$ so that each power in $\pi r T$ cancels out in Eq. (A1). Using Eq. (A4), we find $\left(\frac{r_{+2}}{r}\right)^{d-1}=1+\frac{(d-1) b_{1}}{\pi r T}+\frac{(d-1)\left(b_{2}+\frac{(d-2) b_{1}^{2}}{2}\right)}{(\pi r T)^{2}}$ and $\left(\frac{r_{+2}}{r}\right)^{2}=1+\frac{2 b_{1}}{\pi r T}+\frac{\left(b_{1}^{2}+2 b_{2}\right)}{(\pi r T)^{2}}+\ldots$. Since the terms in $\frac{1}{\pi r T}$ of the $\left(\frac{r_{+1}}{r}\right)^{d-1}$ expansion and the $\left(\frac{d-3}{4 \pi r T}\right)^{2}$ expansion are only dependent on $b_{1}$, and the polynomial Eq. (A1) only has $\frac{1}{(\pi r T)^{2}}$ showing up, the only way for these terms to cancel out is by setting $b_{1}=0$. Then, canceling out the terms in $\frac{1}{(\pi r T)^{2}}$ is done by setting $b_{2}=-\frac{d-3}{16}$. One can now check that the next leading order term is in $\frac{1}{(\pi r T)^{4}}$. With these values for the $b_{i}$, Eq. (A4) is now

$$
r_{+2}=r\left(1-\frac{d-3}{16(\pi r T)^{2}}+\mathcal{O}\left(\frac{1}{(\pi r T)^{4}}\right)\right) .
$$

Equations (A3) and (A5), are precisely the Eqs. (6) and (7), respectively, in the text. 


\section{APPENDIX B: THE PHOTON ORBIT RADIUS $r_{\mathrm{ph}}$ AND THE BUCHDAHL RADIUS $r_{\text {Buch }}$ IN THE $d$-DIMENSIONAL SCHWARZSCHILD SOLUTION}

The Schwarzschild solution was generalized to $d$ dimensions by Tangherlini [22], and it is variously called $d$-dimensional Schwarzschild solution or SchwarzschildTangherlini solution. Here, we have opted to use the first name.

The photon orbit radius, or massless particle otbit radius, appears naturally in the context of particle dynamics in a Schwarzschild $d$-dimensional background. In $d$ spacetime dimensions, it is [23]

$$
r_{\mathrm{ph}}=\left(\frac{d-1}{2}\right)^{\frac{1}{d-3}} r_{+} .
$$

For $d=4$, one gets $r_{\mathrm{ph}}=\frac{3}{2} r_{+}$, a result which is derived in all books in general relativity. This radius is also the radius for a cavity of radius $r$, below which a black hole with horizon radius $r_{+}$in the canonical ensemble is thermodynamically stable. At this radius, $r=r_{\mathrm{ph}}$, the heat capacity $C_{A}$ at constant ensemble area $A$, with $A=\Omega_{d-2} r^{d-2}$ and $\Omega_{d-2}$ being the solid angle in a spherical $d$-dimensional spacetime, is zero, $C_{A}=0$, and for $r<r_{\mathrm{ph}}$, the heat capacity is positive, $C_{A}>0$.

The Buchdahl radius appears naturally in the context of star structure and dynamics in general relativity. It is a bound that states that under some generic conditions for a spherical star of radius $r$, the spacetime is free of singularities for $r_{\text {Buch }} \leq r$. For $r_{\text {Buch }} \geq r$, the star supposedly can collapse into a black hole. In $d$ spacetime dimensions, $r_{\text {Buch }} \geq r$ is [24]

$$
r_{\mathrm{Buch}}=\left(\frac{(d-1)^{2}}{4(d-2)}\right)^{\frac{1}{d-3}} r_{+} .
$$

This radius is as well a limit for spherical thin shells in $d$-dimensional spacetimes that have an equation of state given by $p \leq \frac{1}{d-2} \sigma$, where $\sigma$ is the energy density of the shell and $p$ the tangential pressure on the shell. At the equality, one gets the Buchdahl radius given in Eq. (B2). In such a case, $\sigma=\frac{1}{4 \pi} \frac{d-2}{d-1} \frac{1}{r_{\text {Buch }}}$ and $p=\frac{1}{4 \pi} \frac{1}{d-1} \frac{1}{r_{\text {Buch }}}$. Shells with lesser radius have to have a stiffer equation of state. So, $p \leq \frac{1}{d-2} \sigma$ imposes a Buchdahl bound for shells. For $d=4$, one finds $r_{\mathrm{Buch}}=\frac{9}{8} r_{+}$, a result derived by Buchdahl first for a spherical perfect fluid star [20] and later for thin shells in Ref. [21]. The Buchdahl radius is also the radius that a cavity in the canonical ensemble for spherical gravitation has, such that the free energy $F$ of the system is zero $F=0$, and so above the Buchdahl radius, classical hot flat space does not nucleate into a black hole, and below the Buchdahl radius, classical hot flat space does nucleate into a black hole. It is clear that the two contexts in which $r_{\text {Buch }}$ appears are clearly correlated. That the Buchdahl radius enters into thermodynamics of black holes in the canonical ensemble was noticed first in Ref. [12] where in five dimensions the radius is $r_{\mathrm{ph}}=\frac{2}{\sqrt{3}} r_{+}$.

Thus, the photon orbit radius $r_{\mathrm{ph}}$ and the Buchdahl radius $r_{\text {Buch }}$ appear in two separate contexts, the former in both particle dynamics and in thermodynamics and the latter in both star dynamics and in thermodynamics. The two contexts for $r_{\mathrm{ph}}$, precisely, particle dynamics in a Schwarzschild background on one side and black hole thermodynamic stability on the other, are somehow correlated, although this correlation has not been clearly interpreted. The two contexts for $r_{\text {Buch }}$, namely, general relativistic star solutions and gravitational collapse dynamics on one side and black hole thermodynamic on the other, are, on the other hand, clearly correlated. It also hints that $r_{\text {Buch }}$ is an intrinsic property of the Schwarzschild spacetime, as the radius of the photon orbit, $r_{\mathrm{ph}}$, is. Note also from Eqs. (B1) and (B2) that $\frac{r_{\mathrm{Buch}}}{r_{\mathrm{ph}}}=\left(\frac{1}{2} \frac{d-1}{d-2}\right)^{\frac{1}{d-3}}$. For $d \rightarrow \infty$, one has $r_{\mathrm{Buch}}=r_{\mathrm{ph}}=r_{+}=0$.

\section{APPENDIX C: CONNECTION TO THERMODYNAMICS OF THIN SHELLS IN $\boldsymbol{d}$ SPACETIME DIMENSIONS}

Besides the black hole in the canonical ensemble, another system that can have an exact thermodynamic treatment is provided by spherical thin shells. We compare here the thermodynamics of Schwarzschild black holes and classical hot flat space in a cavity with radius $r$ at a fixed temperature $T$ in the canonical ensemble in $d$ dimensions that we analyzed with the thermodynamics of a selfgravitating Schwarzschild thin shell, i.e., a thin shell with a Minkowski interior and a Schwarzschild exterior, with radius $r$ at a fixed temperature $T$ in $d$ dimensions [18]. These thin matter shells are $(d-2)$-dimensional branes in a spacetime of $d$ dimensions.

In the black hole in the canonical ensemble case, one has a cavity bounded by a massless boundary or massless thin shell, which has radius $r$ and is at temperature $T$. The black hole, when there is one, is inside the boundary, and it has a gravitational or event horizon radius $r_{+}$. There is also the possibility that inside the cavity, there is only hot flat space, which for this purpose is pure hot Minkowski space, i.e., classical hot flat space. In the self-gravitating thin matter shell case, one has that the shell is located at radius $r$ and is at fixed temperature $T$. The shell has rest mass $M$, and so the spacetime has a gravitational radius $r_{+}$, which is not an event horizon radius, since there is no event horizon in this case. The thin shell is a classical object.

Let us analyze the procedures for a black hole in the canonical ensemble in $d$ dimensions and the procedure for the thermodynamic thin matter shell in $d$ dimensions. The procedures are different. The procedure for the black hole in a cavity is through the path integral statistical 
mechanics approach where a gravitational canonical ensemble is defined, which is then used to obtain all the thermodynamic properties, as we have seen here for $d$-dimensional spacetimes.

The procedure for the self-gravitating thin shell is through local thermodynamics alone. The first law of thermodynamics at the thin shell is used. Let us see this; see also Ref. [18] for a thorough analysis of thermodynamics of thin shells in $d$ spacetime dimensions. In the thermodynamic analysis of a Schwarzschild thin matter shell, a spherical static matter shell with rest mass $M$, radius $r$, thus area $A=\Omega_{d-2} r^{d-2}$, and tangential pressure $p$, with a well-defined local temperature $T$, obeys the first law of thermodynamics $T d S=d M+p d A$, where $S$ is its entropy. $T$ and $p$ have to be provided through equations of state, and then the entropy is generically given by $S=S(M, A)$. Using the spacetime general relativity junction conditions, one gets a relation between the gravitational radius $r_{+}$, the proper mass $M$, and $r$, i.e., $r_{+}=r_{+}(M, r)$, and in addition an expression for the tangential pressure $p$ in terms of $M$ and $A$. Another set of conditions besides the junction conditions is the one provided by the integrability conditions for the first law, so that the entropy $S$ is an exact differential. For a Schwarzschild shell there is only one integrability condition. It gives that the local temperature at the shell $T(M, r)$, or $T\left(r_{+}(M, r), r\right)$ if one prefers, must have the Tolman form for the temperature, i.e., $T\left(r_{+}, r\right)=$ $\frac{T_{\infty}\left(r_{+}\right)}{k\left(r_{+}, r\right)}$ where $k\left(r_{+}, r\right)$ is the redshift factor, $k=\sqrt{1-\frac{r_{+}}{r}}$, and $T_{\infty}\left(r_{+}\right)$is a function of $r_{+}$only to be chosen at our will. $T_{\infty}\left(r_{+}\right)$is a free function. Physically, $T_{\infty}\left(r_{+}\right)$can be interpreted as the temperature a small amount of radiation would have at infinity after leaking out from the shell at temperature $T$.

Let us analyze now the results for a black hole in the canonical ensemble in $d$ dimensions and the results for the thermodynamic thin matter shell in $d$ dimensions. The results have many similarities.

First, we analyze and compare the temperatures in each case. For the black hole in the canonical ensemble, the temperature $T$ of a heat bath at the cavity's boundary at radius $r$ is fixed, and since the black hole has mandatorily the Hawking temperature $T_{H}$, this obliges, through the Tolman formula, the black hole radius to be fixed, the computation showing that there are two equilibrium black hole solutions, one large and stable and one small and unstable. For the thermodynamic thin matter shell at radius $r$, one puts it at some fixed temperature $T$, which it is shown to obey the strict Tolman formula, $T\left(r_{+}, r\right)=\frac{T_{\infty}\left(r_{+}\right)}{k\left(r, r_{+}\right)}$, with $T_{\infty}\left(r_{+}\right)$a free function. This free function can be any wellbehaved function of $r_{+}$. In particular, $T_{\infty}\left(r_{+}\right)$can have the Hawking expression $T_{\infty}\left(r_{+}\right)=T_{H}$. In this case, when the thin shell has its temperature at infinity equal to the Hawking temperature, then the two systems, namely, the black hole in the canonical ensemble and the thin shell, are thermodynamically identical in many respects.

Second, we analyze and compare the energies and pressures in each case. For the black hole in the canonical ensemble, the thermodynamic energy $E$ at the cavity's radius $r$ is $E=\frac{(d-2) \Omega_{d-2} r^{d-3}}{8 \pi}\left(1-\sqrt{1-\frac{r_{-}^{d-3}}{r^{d-3}}}\right)$, and so with $r_{+}^{d-3}=\frac{16 \pi}{(d-2) \Omega_{d-2}} m$, one finds that the spacetime mass $m$ is $m=E-\frac{4 \pi E^{2}}{(d-2) \Omega_{d-2} r^{d-3}}$. For the black hole in the canonical ensemble, the pressure $p$, which is a thermodynamic tangential pressure, at the cavity's radius $r$, is $p=\frac{d-3}{16 \pi r \sqrt{1-\frac{r^{d-3}}{r^{d-3}}}}\left(1-\sqrt{1-\frac{r_{+}^{d-3}}{r^{d-3}}}\right)^{2}$. For the thin matter shell at radius $r$, assumed to be composed of a perfect fluid, one has to find its stress-energy tensor $S_{a b}$, where $a, b$ are spacetime indices on the shell. $S_{a b}$ can be put in diagonal form, and its components are characterized by the rest mass energy density $\sigma$ and the tangential pressure $p$ acting on a $(d-2)$-sphere at radius $r$. The junction conditions give that the rest mass energy density $\sigma$ is $\sigma=\frac{(d-2)}{8 \pi r}\left(1-\sqrt{1-\frac{r_{r}^{d-3}}{r^{d-3}}}\right)$. Since the rest mass $M$ of the shell is given by $M=\sigma A$ with $A=\Omega_{d-2} r^{d-2}$, the rest mass is $M=\frac{(d-2) \Omega_{d-2} r^{d-3}}{8 \pi}\left(1-\sqrt{1-\frac{r_{+}^{d-3}}{r^{d-3}}}\right)$. Putting $r_{+}^{d-3}=$ $\frac{16 \pi}{(d-2) \Omega_{d-2}} m$ into the expression for $M$, one finds that the spacetime mass $m$ is $m=M-\frac{4 \pi M^{2}}{(d-2) \Omega_{d-2} r^{d-3}}$. For the thin matter shell at radius $r$, the pressure $p$, which is a dynamical tangential pressure derived from the junction conditions, is $p=\frac{d-3}{16 \pi r \sqrt{1-\frac{r^{d-3}}{r^{d-3}}}}\left(1-\sqrt{1-\frac{r_{+}^{d-3}}{r^{d-3}}}\right)^{2}$. Clearly, the thermodynamic energy $E$ in the black hole case and the rest mass $M$ in the thin shell case have the same expression and so can be identified, i.e., $E=M$. $E$ and $M$ are quasilocal energies. Also, clearly, the thermodynamic pressure $p$ in the black hole case and the dynamical pressure $p$ in the thin shell have the same expression and so can be identified.

Third, we analyze and compare the entropies in each case. For the black hole in the canonical ensemble, the entropy is the Bekenstein-Hawking area law $S=\frac{1}{4} A_{+}$, for both the stable and the unstable black holes. For the thermodynamic thin matter shell, one finds that for any well-behaved $T_{\infty}\left(r_{+}\right)$, its entropy is given by a function of $r_{+}$alone, $S=S\left(r_{+}\right)$, independent of the shell radius $r$. In particular, when the shell is put at a temperature $T$ such that the temperature at infinity is the Hawking temperature $T_{\infty}\left(r_{+}\right)=T_{H}$, then the entropy of the shell $S=S\left(r_{+}\right)$is definitely given by the Bekenstein-Hawking area law $S=\frac{1}{4} A_{+}$. Moreover, when the shell is at $r_{+}, r=r_{+}$, then the temperature at infinity has to be mandatorily the Hawking temperature; otherwise, quantum effects render the whole system unstable and undefined. Thus, 
when the shell turns into a black hole, more properly into a quasiblack hole, one recovers from the shell thermodynamics the black hole's expressions.

Fourth, we analyze and compare the thermal stability in each case. For the black hole in the canonical ensemble, the heat capacity $C_{A}$ is the quantity that signals thermodynamic stability if $C_{A} \geq 0$ from thermodynamic instability if $C_{A}<0$. It was shown that $C_{A}=$ $\frac{(d-2)}{2(d-1)} \Omega_{d-2} r_{+} r^{d-3} \frac{1-\frac{r_{-}^{d-3}}{r^{d-3}}}{1-\frac{2}{d-1-3}}$, and it implies that when the cavity's radius $r$ is less than or equal to the radius of the circular photon orbits, i.e., $r_{+}<r \leq r_{\text {ph }}$, the black hole is thermodynamically stable, otherwise unstable, this meaning that the large black hole $r_{+2}$ is the stable one and the smaller $r_{+1}$ is unstable. For the thin matter shell, there is also the thermodynamic stability criterion $C_{A} \geq 0$ as well as other stability criteria which further restrict the thermodynamic stability. The particular interesting case, the one related to the black hole in the canonical ensemble, is when the temperature of the shell at infinity is the Hawking temperature $T_{H}$. In this very case, the heat capacity $C_{A}$ has the expression $C_{A}=\frac{(d-2)}{2(d-1)} \Omega_{d-2} r_{+} r^{d-3} \frac{1-\frac{r^{d-3}}{r^{d-3}}}{1-\frac{2}{d-1-3} \text {, }}$, and so for stability, the self-gravitating matter shell must be placed between its own gravitational and its photon sphere for stability, i.e., $r_{+}<r \leq r_{\mathrm{ph}}$. Thus, in the case that the temperature of the shell at infinity is the Hawking temperature, and so in the situation that is thermodynamic similar to the black hole, the thermodynamic criterion of positive heat capacity gives the same result for both systems.

Fifth, we analyze and compare the generalized free energy function in each case. For the black hole in the canonical ensemble, the free energy $F$ gives a special cavity radius $r$ for which it is zero. This radius is the Buchdahl radius $r_{\text {Buch }}$ that appears naturally in general relativistic star structure and dynamics, especially in star gravitational collapse. It also appears in the black hole thermodynamic context. For $r \geq r_{\text {Buch }}$ classical hot flat space that does transition to a black hole, for $r<r_{\text {Buch }}$, there is a phase transition from classical hot flat space to a black hole. For the thin matter shell, with the identification of the mass $M$ with the thermal energy $E, M=E$, a free energy $F$ can be defined by $F=M-T S$. When the temperature of the shell at infinity is the Hawking temperature $T_{H}$ and thus the shell has the Bekenstein-Hawking entropy, such a free energy $F$ also gives the Buchdahl radius $r_{\text {Buch }}$ as the special cavity radius $r$ for which $F=0$, presumably meaning that it is energetically favorable for the shell to disperse away at this radius in the given conditions. Here, the Buchdahl radius $r_{\text {Buch }}$ appears also as a structure and dynamic radius on top of being a thermodynamic one. Indeed, by imposing that the equation of state for the matter in the shell obeys $p \leq \frac{1}{d-2} \sigma$, i.e., by imposing that the pressure is equal to or less than the radiation pressure, a sort of energy condition, specifically, the trace of the stress-energy tensor $S_{a b}$ is equal to or less than zero, $\operatorname{Tr} S_{a b} \leq 0$, one finds that the bound $p=\frac{1}{d-2} \sigma$, is satisfied for the $d$-dimensional Buchdahl radius, i.e., $r_{\text {Buch }}=\left(\frac{(d-1)^{2}}{4(d-2)}\right)^{\frac{1}{d-3}} r_{+}$. Shells with lesser radius have to have a stiffer equation of state. So, $p \leq \frac{1}{d-2} \sigma$ imposes a Buchdahl bound for shells. Thus, one finds that the free energy in both cases is zero when the radius of the cavity $r$ or the radius of the shell $r$ are at the Buchdahl radius, in the latter case meaning that the pressure at the shell is equal to the radiation pressure.

Thus, this thorough comparison between the black hole in the canonical ensemble in $d$ dimensions and the thin matter shell in $d$ dimensions shows that indeed, when the situations are similar, explicitly, when the shell's temperature at infinity is the Hawking temperature, and for the quantities that it makes sense to perform a comparison, the two systems behave thermodynamically in similar ways. The boundary of the black hole cavity at a definite temperature defines a heat reservoir; analogously, the shell at a definite temperature is a heat reservoir.

\section{APPENDIX D: QUANTUM HOT FLAT SPACE IN $d$ SPACETIME DIMENSIONS}

The first law of thermodynamics for quantum hot flat space is written as

$$
T d S_{\mathrm{HFS}}=d E_{\mathrm{HFS}}+P_{\mathrm{HFS}} d V,
$$

where $T$ is the temperature of the space, $S_{\mathrm{HFS}}$ is the quantum hot flat space entropy, $E_{\mathrm{HFS}}$ is its internal energy, $P_{\mathrm{HFS}}$ is its radiation pressure, and $V$ is the volume it occupies. The internal energy $E_{\mathrm{HFS}}$ of such a radiation gas has the usual formula, definitely,

$$
E_{\mathrm{HFS}}=N V a T^{d},
$$

where $N$ is the total number of massless states and $a$ is a quantum mechanics constant given by $a=\frac{\Gamma(d) \zeta(d)}{2^{d-2} \pi^{\frac{d-1}{2}} \Gamma\left(\frac{d-1}{2}\right)}$, with $\Gamma$ and $\zeta$ being the gamma and zeta functions, respectively. The constant $a$ is related to the $d$-dimensional Stefan-Boltzmann constant $\sigma$ through $\sigma=a \frac{\sqrt{\pi} \Gamma\left(\frac{d}{2}\right)}{2 \Gamma\left(\frac{d-1}{2}\right)}$ [25], which for $d=4$ simplifies to $\sigma=a$. The equation of state for radiation that gives a relation between the radiation pressure $P_{\mathrm{HFS}}, V$, and $E_{\mathrm{HFS}}$, is

$$
P_{\mathrm{HFS}} V=\frac{1}{d-1} E_{\mathrm{HFS}},
$$

so that using Eq. (D2), one finds $P_{\mathrm{HFS}}=\frac{N a}{d-1} T^{d}$. From the first law Eq. (D1) and using Eqs. (D2) and (D3), one finds the entropy of quantum hot flat space 


$$
S_{\mathrm{HFS}}=\frac{d}{d-1} \frac{E_{\mathrm{HFS}}}{T}
$$

i.e., $S_{\mathrm{HFS}}=\frac{d}{d-1} N V a T^{d-1}$. The free energy for quantum hot flat space is

$$
F_{\mathrm{HFS}}=E_{\mathrm{HFS}}-T S_{\mathrm{HFS}} .
$$

From Eq. (D4) on Eq. (D5), one has

$$
F_{\mathrm{HFS}}=-\frac{1}{d-1} E_{\mathrm{HFS}},
$$

and using Eq. (D2), one finds $F_{\mathrm{HFS}}=-\frac{1}{d-1} N V a T^{d}$. Now, the volume $V$ of a spherical cavity in $d$ dimensions is $V=\frac{\Omega_{d-2}}{d-1} r^{d-1}$, with $\Omega_{d-2}=\frac{2 \pi^{\frac{d-1}{2}}}{\Gamma\left(\frac{d-1}{2}\right)}$ being the solid angle in spherical $d$-dimensional spacetime. We have not specified yet the number of degrees of freedom $N$, and as it is, the expression works for any radiation gas of massless particles in flat space at finite temperature in $d$ dimensions. If there are only gravitons within the cavity, $N$ is given by $N=\frac{d(d-3)}{2}$. For this $N$, Eq. (D6) together with Eq. (D2) gives

$$
F_{\mathrm{HFS}}=-\frac{d(d-3)}{2(d-1)^{2}} a \Omega_{d-2} r^{d-1} T^{d},
$$

which is the expression we use in Eq. (36). To complete, since $I=\beta F$ and $\beta=\frac{1}{T}$, the action for $d$-dimensional quantum hot flat space is

$$
I_{\mathrm{HFS}}=-\frac{d(d-3)}{2(d-1)^{2}} a \Omega_{d-2} r^{d-1} T^{d-1} .
$$

\section{APPENDIX E: CLASSICAL HOT FLAT SPACE IN $d$ SPACETIME DIMENSIONS AS A PRODUCT OF QUANTUM HOT FLAT SPACE AND THE CORRESPONDING BLACK HOLE PHASE TRANSITIONS}

It is of interest to understand the passage to classical hot flat space in $d$ spacetime dimensions from quantum hot flat space and look into the black hole phase transitions from classical hot flat space in some more detail.

In classical hot flat space, one puts $a=0$, and so Eq. (36), or Eq. (D7), reads now

$$
F_{\mathrm{HFS}}=0 .
$$

Thus, Eq. (38), which states the condition for the stable black hole $r_{+2}$ to be the ground state, turns into

$$
F\left(r_{+2}\right) \leq 0 \text {. }
$$
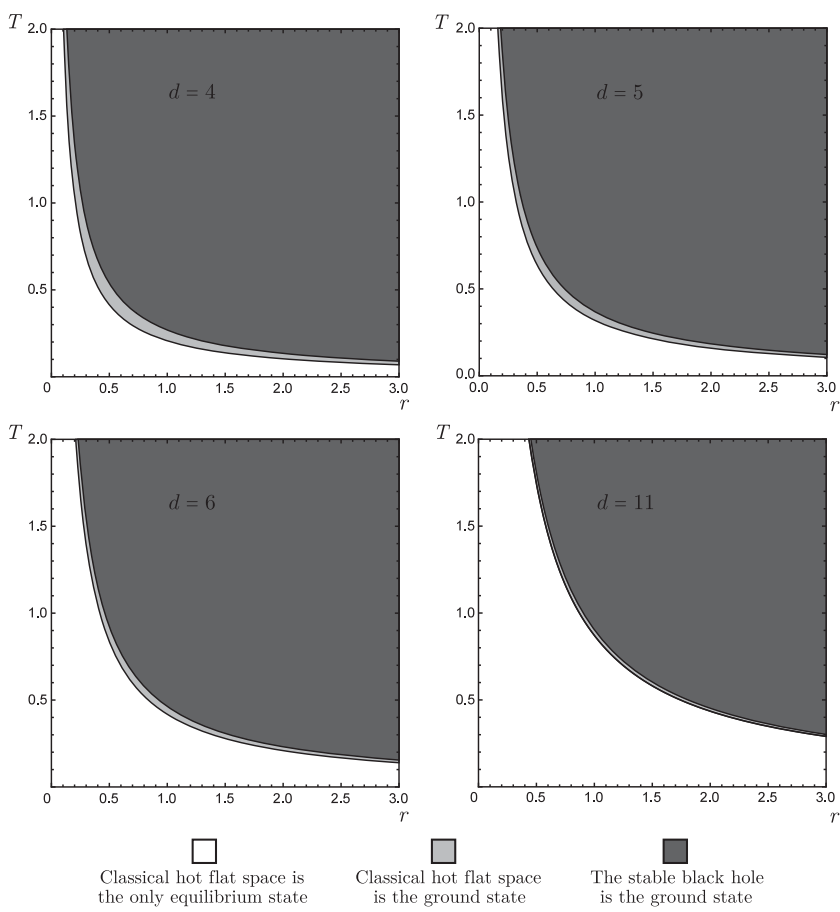

FIG. 6. Phase diagram of the gravitational canonical ensemble in the classical hot flat space case with a plot of the cavity radius $r$, in arbitrary units, versus the temperature $T$, in arbitrary units, for four different dimensions, $d=4,5,6$, and 11. Each point in the plots represents a different configuration of the ensemble. Each plot is separated in three different regions. The white region in each plot, delimited by the hyperbola $\pi r T \geq \frac{d-3}{4}\left[\left(\frac{2}{d-1}\right)^{\frac{2}{d-3}}-\left(\frac{2}{d-1}\right)^{\frac{d-1}{d-3}}\right]^{-1 / 2}$, see Eq. (5), is the region where in thermodynamic equilibrium there is only classical hot flat space, with no black holes. Each point in the hyperbola gives the photon radius $r_{\mathrm{ph}}$ and the corresponding temperature. The gray region in each plot is characterized by having as the ground state classical hot flat space and is delimited also by the other hyperbola $\pi r T=\left(\frac{(d-1)^{d-1}}{4^{d-2}(d-2)}\right)^{\frac{1}{d-3}}$; see Eq. (34). Each point in this hyperbola gives the Buchdahl radius $r_{\mathrm{Buch}}$ and the corresponding temperature. The dark region in each plot, delimited by the latter hyperbola, is characterized by having as the ground state the larger stable black hole $r_{+2}$. The thick black line in each plot yields a mixed phase, i.e., a superposition of the classical hot flat space ground state phase with the stable black holes ground state phase. Comparing this figure with Fig. 3 for quantum hot flat space, one sees that classical hot flat space approximates quantum hot flat space for large cavity radius $r$ and low temperature $T$.

The phase diagram for black holes and a classical hot flat space in $d=4,5,6$, and 11 dimensions is now given in Fig. 6 , which is the limit of Fig. 3 when $a=0$.

Equation (E2) has no extrema, unlike the quantum case of Eq. (38). Using Eq. (38), we have found a minimum radius $r_{\min }$ such that if the cavity's radius $r$ obeys $r<r_{\min }$, only quantum hot flat space can be the ground state. We have also found, using Eq. (38), a maximum temperature $T_{\max }$ such that if the cavity's temperature $T$ obeys 
$T>T_{\max }$, only quantum hot flat space could be the ground state. For classical hot flat space, these extrema do not occur, or to be more precise, one finds $r_{\min }=0$ and $T_{\max }=\infty$.

Since $r_{\min }$ and $T_{\max }$ do not enter the problem if one uses classical hot flat space, the only parameter that matters is $\pi r T$; see Eq. (34) or (35). Thus, here there are two important hyperbolas when working out the phase diagram for classical hot flat space. There is the hyperbola $\pi r T=\frac{d-3}{4}\left[\left(\frac{2}{d-1}\right)^{\frac{2}{d-3}}-\left(\frac{2}{d-1}\right)^{\frac{d-1}{d-3}}\right]^{-1 / 2}$, see Eq. (5), that separates the region where classical hot flat space is the only equilibrium state from the region where classical hot flat space is the ground state. As we have seen, each point in this hyperbola gives the photon radius $r_{\mathrm{ph}}=$ $\left(\frac{d-1}{2}\right)^{\frac{1}{d-3}} r_{+}$for the radius of the cavity and the corresponding cavity's temperature. There is the other hyperbola $\pi r T=\left(\frac{(d-1)^{d-1}}{4^{d-2}(d-2)}\right)^{\frac{1}{d-3}}$, see Eq. (34), that separates the region where classical hot flat space is the ground state from the region where stable black holes $r_{+2}$ is the ground state and so classical hot flat space can nucleate stable black holes $r_{+2}$. Each point in this hyperbola, characterized by a specific cavity radius and a specific cavity's temperature, corresponds to a specific thermodynamic system, and, as we have seen, each such point also yields the Buchdahl radius, $\quad r_{\mathrm{Buch}}=\left(\frac{(d-1)^{2}}{4(d-2)}\right)^{\frac{1}{d-3}} r_{+}$, for the system under consideration.

Thus, there are three phases. One phase when the cavity's radius $r$ and the cavity's temperature $T$ only give the possibility of the existence of classical hot flat space, there are no stable equilibrium black holes $r_{+2}$, and for that matter also no unstable equilibrium black holes $r_{+1}$, but eventually black holes out of thermodynamic equilibrium may appear in this phase. Another phase is when the cavity's radius $r$ obeys $r_{\text {Buch }}<r<r_{\text {ph }}$, where classical hot flat space is the ground state and so stable black holes $r_{+2}$ can transition into classical hot flat space. And yet another phase is when the cavity's radius $r$ obeys $r<r_{\text {Buch }}$ where the stable black hole $r_{+2}$ is the ground state and so classical hot flat space can nucleate stable black holes $r_{+2}$. There is also a mixed phase, which is a superposition of the two previous phases. In Fig. 6, the three phases are represented by the white, gray, and dark regions, respectively, and the mixed phase is represented by a thick black line between the gray and dark regions.

Comparing Fig. 6 for classical hot flat space with Fig. 3 for quantum hot flat space, one sees that classical hot flat space approximates quantum hot flat space for large cavity radius $r$ and low temperature $T$. Two important consequences can be drawn from this comparison. One consequence is that in classical hot flat space, as the number of spacetime dimensions increases, the region for which stable black holes can transition into classical hot flat space gets smaller, whereas the region for which classical hot flat space can nucleate stable black holes gets larger, contrarily to what happens in quantum hot flat space, thus showing clearly that the classical approximation is not valid for a vast region of the $r \times T$ plane. The other consequence of this comparison is that the Buchdahl radius is an important radius in the classical approximation, as one would expect.

\section{APPENDIX F: SYNOPSIS AND ADDITIONS}

The canonical ensemble statistical mechanics formalism has been used for $d$-dimensional black holes, and the corresponding thermodynamics has been studied in detail, thereby extending York's four-dimensional results and previous five-dimensional results.

When working out the possible black hole radii for a given cavity radius $r$ and a given cavity temperature $T$, one finds that in four dimensions one needs to solve a cubic equation and in five dimensions one needs to solve a quartic that can be reduced to a quadratic equation. In both cases, the solutions yield two real radii yielding the two black holes. In $d$ dimensions, one has a polynomial equation of order $d-1$, and one could expect a different number of real solutions, but there are also only two, with radii $r_{+1}$ and $r_{+2}$, yielding again two possible black holes.

The formalism shows that for any dimension $d$, the two black holes in equilibrium, the small $r_{+1}$ and the large $r_{+2}$, both have a Bekenstein-Hawking entropy, although the procedure is only well defined for the larger one since it is stable and the laws of thermodynamics can be applied, whereas the smaller one is unstable and cannot be treated properly through thermodynamics.

There are two distinct characteristic radii that appear automatically naturally in the canonical black hole thermodynamics. One characteristic radius is the photon sphere radius $r_{\mathrm{ph}}$. The photon sphere radius that appears in the Schwarzschild geometry, particularly when there is a black hole, is the radius for which any massless particle, including photons, can have circular orbits. Notably, in $d$ dimensions, for a given temperature $T$ of the heat bath, the photon sphere radius also appears as separating black hole systems that are thermodynamically stable from black hole systems that are unstable. Being a characteristic that seems to appear in any dimension $d$, this shows that there is some intrinsic property of the photon sphere location that connects it to thermodynamic stability. However, a full explanation has not been given yet. The other characteristic radius that appears automatically in these canonical ensemble thermodynamic systems is the Buchdahl radius $r_{\text {Buch }}$. The Buchdahl radius is the minimum radius that, under certain general conditions, a spherically symmetric interior matter solution with Schwarzschild exterior can have. Surprisingly, this radius also shows up in the canonical ensemble setting, as the radius for which the free energy of the stable black hole $r_{+2}$ passes through zero, as we have shown. This happens in any dimension $d$. Since the two occasions at which $r_{\text {Buch }}$ emerges are totally distinct, it seems that $r_{\text {Buch }}$ also signals 
some intrinsic property of the spacetime geometry. Indeed, $r_{\text {Buch }}$ is also related to thin shells, where it appears both for dynamic and for thermodynamic reasons.

The formalism also permits comparing the free energy of $d$-dimensional quantum hot flat space with the free energy of the $d$-dimensional stable black hole $r_{+2}$ and thereby identify the conditions for which the ground state of the canonical ensemble is quantum hot flat space or the stable black hole. It was found that for sufficiently low cavity temperatures $T$ and sufficiently large cavity radius $r$, in Planck units, the ground state is the stable black hole phase, but in the limit of a very high number of dimensions $d$, this phase gets vanishing small, and so in this limit black holes never nucleate.
[1] J. B. Hartle and S. W. Hawking, Path-integral derivation of black-hole radiance, Phys. Rev. D 13, 2188 (1976).

[2] S. W. Hawking, The path-integral approach to quantum gravity, in General Relativity: An Einstein Centenary Survey, edited by S. W. Hawking and W. Israel (Cambridge University Press, Cambridge, England, 1979), p. 746.

[3] M. Hewitt, Vacuum polarisation on higher dimensional black hole spacetimes, Ph.D. thesis, University of Sheffield, 2015, http://etheses.whiterose.ac.uk/8342/.

[4] M. Bañados, C. Teitelboim, and J. Zanelli, Black Hole Entropy and the Dimensional Continuation of the GaussBonnet Theorem, Phys. Rev. Lett. 72, 957 (1994).

[5] J. W. York, Black hole thermodynamics and the Euclidean Einstein action, Phys. Rev. D 33, 2092 (1986).

[6] D. J. Gross, M. J. Perry, and L. G. Yaffe, Instability of flat space at finite temperature, Phys. Rev. D 25, 330 (1982).

[7] B. Allen, Euclidean Schwarzschild negative mode, Phys. Rev. D 30, 1153 (1984).

[8] H. W. Braden, J. D. Brown, B. F. Whiting, and J. W. York, Charged black hole in a grand canonical ensemble, Phys. Rev. D 42, 3376 (1990).

[9] C. S. Peça and J. P. S. Lemos, Thermodynamics of ReissnerNordström-anti-de Sitter black holes in the grand canonical ensemble, Phys. Rev. D 59, 124007 (1999).

[10] O. B. Zaslavskii, Canonical ensemble for arbitrary configurations of self-gravitating systems, Phys. Lett. A 152, 463 (1991).

[11] E. A. Martinez and J. W. York, Additivity of the entropies of black holes and matter in equilibrium, Phys. Rev. D 40, 2124 (1989).

[12] R. André and J.P. S. Lemos, Thermodynamics of fivedimensional Schwarzschild black holes in the canonical ensemble, Phys. Rev. D 102, 024006 (2020).

[13] J. P. Gregory and S. F. Ross, Stability and the negative mode for a Schwarzschild black hole in a finite cavity, Phys. Rev. D 64, 124006 (2001).
[14] H. S. Reall Classical and thermodynamic stability of black branes, Phys. Rev. D 64, 044005 (2001).

[15] J. X. Lu, S. Roy, and Z. Xiao, Phase transitions and critical behavior of black branes in canonical ensemble, J. High Energy Phys. 01 (2011) 133.

[16] E. A. Martinez, Fundamental thermodynamical equation of a self-gravitating system, Phys. Rev. D 53, 7062 (1996).

[17] J.P.S. Lemos, G. M. Quinta, and O. B. Zaslavskii, Entropy of a self-gravitating electrically charged thin shell and the black hole limit, Phys. Rev. D 91, 104027 (2015).

[18] R. André, J. P. S. Lemos, and G. M. Quinta, Thermodynamics and entropy of self-gravitating matter shells and black holes in $d$ dimensions, Phys. Rev. D 99, 125013 (2019).

[19] J. D. Brown and J. W. York, Quasilocal energy and conserved charges derived from the gravitational action, Phys. Rev. D 47, 1407 (1993).

[20] H. A. Buchdahl, General relativistic fluid spheres, Phys. Rev. 116, 1027 (1959).

[21] H. Andréasson, On the Buchdahl inequality for spherically symmetric static shells, Commun. Math. Phys. 274, 399 (2007).

[22] F. R. Tangherlini, Schwarzschild field in $n$ dimensions and the dimensionality of space problem, Nuovo Cimento 27, 636 (1963).

[23] F. C. P. Monteiro, General and specific results for d-dimensional orbits in general relativity: From a fourdimensional spacetime to the large $d$ limit, M.Sc. thesis, Instituto Superior Técnico, Lisbon, 2015.

[24] M. Wright, Buchdahl type inequalities in $d$ dimensions, Classical Quant. Grav. 32, 215005 (2015).

[25] P. T. Landsberg and A. De Vos, The Stefan-Boltzmann constant in $n$-dimensional space, J. Phys. A 22, 1073 (1989). 